\title{
Threatened and uncommon plants of New Zealand
}

\section{P. J. de LANGE}

Science \& Research Unit

Department of Conservation

Private Bag 68908

Newton

Auckland, New Zealand

D. A. NORTON

Conservation Research Group

School of Forestry

University of Canterbury

Private Bag 4800

Christchurch, New Zealand

\section{P. B. HEENAN}

Landcare Research

P. O. Box 69

Lincoln 8152, New Zealand

\section{S. P. COURTNEY}

Department of Conservation

Nelson/Marlborough Conservancy

Private Bag

Nelson, New Zealand

B. P. J. MOLLOY

Research Associate

Landcare Research

P. O. Box 69

Lincoln 1852, New Zealand

C. C. OGLE

22 Forres Street

Wanganui, New Zealand

B. D. RANCE

Department of Conservation

Southland Conservancy

Private Bag

Invercargill, New Zealand

B03016; Online publication date 30 March 2004

Received 13 May 2003; accepted 3 December 2003

\author{
P. N. JOHNSON \\ Landcare Research \\ Private Bag 1930 \\ Dunedin, New Zealand \\ R. HITCHMOUGH \\ Biodiversity Recovery Unit \\ P.O. Box 10420 \\ Wellington, New Zealand
}

\begin{abstract}
A reappraisal of the conservation status of the indigenous New Zealand vascular plant flora is presented. The list comprises 792 taxa (34\% of New Zealand's total indigenous vascular flora) in the following categories: Extinct 4 taxa, Acutely Threatened 122 taxa (comprising 47 taxa Nationally Critical, 54 Nationally Endangered, 21 Nationally Vulnerable), Chronically Threatened 96 taxa (comprising Serious Decline 26 taxa, Gradual Decline 70 taxa), At Risk 499 taxa (comprising Sparse 126 taxa, Range Restricted 373 taxa), Non-resident Native 26 taxa (comprising Vagrant 16 taxa, Colonist 10 taxa), and Data Deficient 45 taxa. A further 208 plants are listed as Taxonomically Indeterminate, being those which might warrant further conservation attention once their taxonomic status is clarified. A further 31 named taxa and 18 rated as Taxonomically Indeterminate, and previously considered to be threatened and/or uncommon, are removed from this updated listing. A concordance of plant names is provided. The lists presented use a new threat classification system developed by the New Zealand Department of Conservation for sole use within this country. This paper represents the first time the entire known indigenous vascular flora has been assessed from a conservation perspective since the mid 1970s. A brief analysis of the patterns of rarity exhibited by the taxa listed is presented.
\end{abstract}

Keywords New Zealand; threatened vascular plants; uncommon vascular plants; conservation status; rarity 


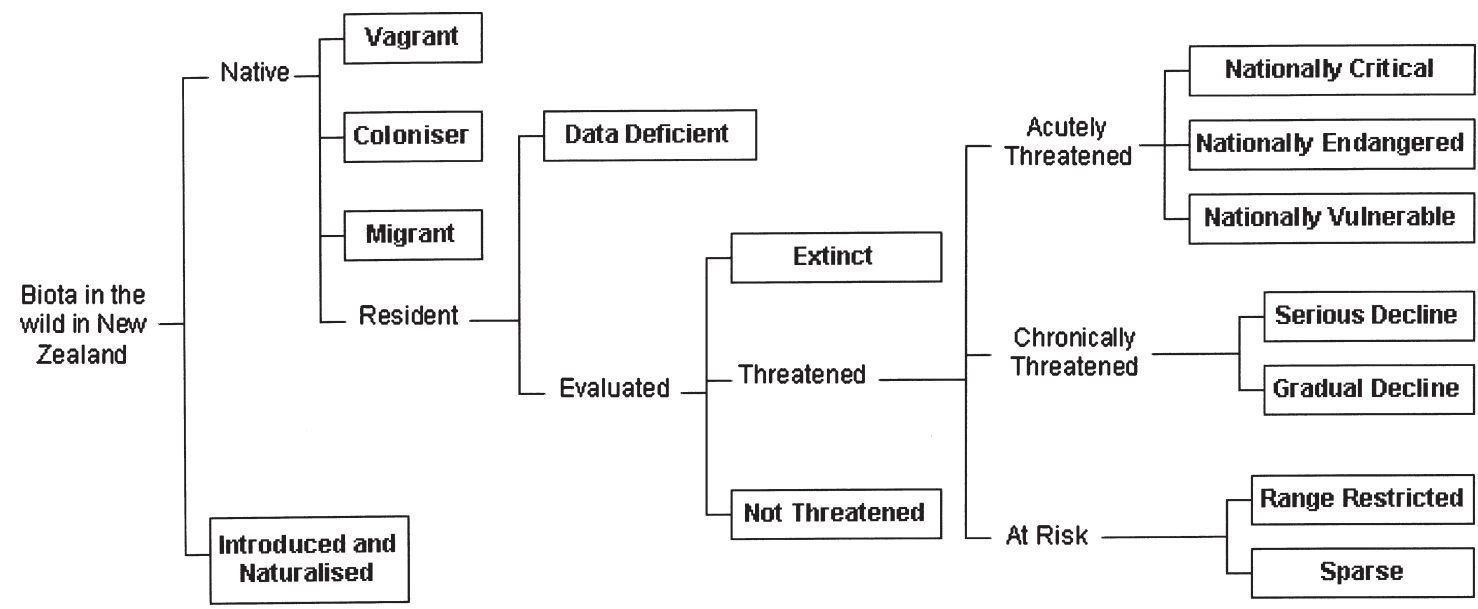

Fig. 1 New Zealand threat classification system (after Molloy et al. 2002). Box denotes a category in the classification.

\section{INTRODUCTION}

The last listing of New Zealand's threatened and uncommon vascular plants was conducted by the New Zealand Threatened Plant Committee in the late 1990s (de Lange et al. 1999). Since that publication, the listing process used has been superseded by the development of a new threat classification system for all New Zealand taxa (Molloy et al. 2002). This system is a refinement of the threat classification framework proposed by de Lange \& Norton (1998) and used by the former New Zealand Threatened Plant Committee for vascular plants, and the Species Priority Ranking System developed and used by the New Zealand Department of Conservation for a range of taxonomic groups (Molloy \& Davis 1992). The new threat classification system, developed by a specialist panel for the Department of Conservation between March 1999 and December 2001 (Molloy et al. 2002), is a uniquely New Zealandbased conservation status assessment tool (cf. IUCN 1994, 2000), which has now been applied for birds, bats, marine mammals, frogs, reptiles, freshwater and marine fish, freshwater, marine, and terrestrial invertebrates, bryophytes, macro-algae, fungi, and vascular plants indigenous to New Zealand (Hitchmough 2002).

As part of the development of the new system, a new panel undertook a comprehensive re-evaluation of the threatened status of the New Zealand vascular flora between October and December 2001 (Hitchmough 2002). This panel comprised some members of the former New Zealand Threatened Plant committee (de Lange et al. 1999) plus appointed representatives from the Department of Conservation, universities, and crown research institutes (see authorship list for this paper). In accordance with agreements reached between the New Zealand Botanical Society Committee, which was responsible for the functions of the former New Zealand Threatened Plant Committee, and the Department of Conservation (Rogan 2002), this paper continues the process of reporting, through peer-reviewed literature, the results of this assessment.

This new list updates and supersedes all previous threatened vascular plant listings (Cameron et al. 1993, 1995; de Lange et al. 1999; Hitchmough 2002) for New Zealand and remains valid from the date of publication until the next list is published.

\section{METHODS}

The following lists are for vascular plant taxa (species, subspecies, varieties, forma, and those entities as yet without formal taxonomic rank) believed to be indigenous to the New Zealand Botanical Region (Wardle 1991), excepting those endemic to Macquarie Island*. Included are indigenous New Zealand plants that also occur naturally overseas (e.g., in Australia), where they may or may

Macquarie Island*, though biogeographically part of the New Zealand subcontinent, is under the control of Australia, thus, New Zealand-based assessments of its biota are unnecessary. 
not be considered threatened (e.g., Amphibromus fluitans, Sebaea ovata). Initial information for this revision was gathered from the previous three listings of threatened, local, and uncommon plants (Cameron et al. 1993, 1995; de Lange et al. 1999), and Flora of New Zealand volumes I-V. This information was supplemented by public submissions and expert opinion regarding the conservation status of the New Zealand indigenous flora. Submissions were solicited from the New Zealand botanical community through the New Zealand Botanical Society Newsletter (Hitchmough 2001). Submissions were then collated and reviewed by the vascular plant panel in October 2001.

The placement of candidate taxa in risk categories was based on the criteria outlined by Molloy et al. (2002), panel knowledge, and referral to herbarium records and recent publications. In situations of doubt, provisional assessments of candidate taxa were referred to specialists in species autecology, reproductive biology, demography, historical and current range information, threats, and projected decline patterns, for their advice. All provisional assessments were then re-evaluated by the panel in December 2001, and the interim listings were posted for external comment by Department of Conservation botanists and those who provided the candidate taxa for the listing process. The lists stemming from that exercise were published by the Department of Conservation (Hitchmough 2002). However, as a result of the incomplete listings in that document, new information, further public comment, and our requirement to publish a paper within peer-reviewed literature (Rogan 2002), the entire vascular plant list of Hitchmough (2002) was reassessed by the panel during January and February 2003.

The risk categories used (Appendices 1 and 2) are those defined in Molloy et al. (2002) (Fig. 1), namely "Extinct", "Acutely Threatened" (Nationally Critical, Nationally Endangered, Nationally Vulnerable), "Chronically Threatened" (Serious Decline, Gradual Decline), "At Risk" (Sparse, Range Restricted), "Non-resident Native" (Coloniser, Vagrant), and "Data Deficient". However, the definition of "Range Restricted" departs from that offered by Molloy et al. (2002) in that an area of $100 \mathrm{~km}^{2}$ is used to spatially define the category.

These categories were preferred by Molloy et al. (2002) over the current IUCN (2000) categories because they reflect more accurately the nature of insular rarity as occurs in New Zealand (cf. de Lange \& Norton 1998). However, as indicated by Molloy et al. (2002), the New Zealand Threat Classification
System does not preclude individuals from using IUCN Threat Categories, and information used for the New Zealand listings presented here, and held by the Department of Conservation, is available to those wishing to undertake an independent IUCN threat assessment.

Unlike earlier listings, except perhaps that of Given (1976), the present listing has resulted from an assessment of all taxa known to be indigenous to New Zealand. Thus, the taxa listed here are the subset of the entire vascular flora that meet the criteria that define the categories of Extinct, Acutely Threatened, Chronically Threatened, At Risk, Non-resident Native, and Data Deficient (Molloy et al. 2002).

Four lists are presented here (Appendices 1-4). Appendix 1 comprises the main New Zealand Threatened and Uncommon Vascular Plant list. Appendix 2 deals with all Taxonomically Indeterminate plants. Appendix 3 lists the vascular plant taxa no longer considered to be at risk or nationally uncommon, or, in the case of unnamed entities, those whose identity could not be determined by experts and which could not be linked to herbarium specimens. Appendix 4 provides a concordance of names used by de Lange et al. (1999) and altered in this publication. Plants listed in Appendix 2 as Taxonomically Indeterminate are assigned a provisional conservation status using the same criteria as in Appendix 1 but recognising that information on their taxonomic relationships has either not been formally evaluated or remains in doubt.

Authority abbreviations of all published plant names follow those recommended by Brummitt \& Powell (1992). Those plants considered to be Taxonomically Indeterminate (212 taxa) are listed by showing their probable affinity (e.g., Ranunculus aff. stylosus) and, where this is not known or there is a suspected aggregate, names are then listed alphabetically (e.g., Cardamine (a) or Lepidium aff. oleraceum (a), (b), et seq.). All plants accepted in the Taxonomically Indeterminate category are supported with a herbarium voucher. Treatment of families follows Kubitzki (1990) for pteridophytes and gymnosperms, and the recommendations of APG II (2003) for angiosperms. The treatment of genera and species is based on recent publications and the opinions of specialists.

A brief analysis of the lists is also presented. To simplify the interpretations we have combined the At Risk and Non-resident Native categories, referring to these collectively as Naturally Uncommon (although some At Risk taxa have that status as a result of past human activities). For the construction 
of some tables we have based our assessments as follows.

Altitudinal zones are based on Wardle (1991) except that we have used "Lowland" to refer to Wardle's "Warm Temperate" zone, and have included Wardle's "Penalpine" and "Nival" zones within our "Alpine" zone. We have also distinguished a coastal zone to refer to those habitats that are exposed to regular influence from the sea as characterised by high saline inputs. The altitudinal zones decrease with increasing latitude so that Campbell Island only has Subalpine and Alpine zones above the Coastal zone.

The habitat types used in the analysis have been adapted from Wardle (1991) and reflect the major physiognomic cover types dominated by indigenous species. "Other scrub" includes scrub communities dominated by Kunzea and Leptospermum species, and open seral communities such as gumlands. "Tall-tussock grassland" includes those grassland communities dominated by Chionochloa and tall tussock-forming Poa species (e.g., Poa foliosa). "Short-tussock grassland" includes those grassland communities dominated by Festuca species, Rytidosperma species, and some Poa species (e.g., Poa cita and $P$. colensoi). "Beach" includes dune systems, and sand, gravel, and boulder beaches.

Individual taxa have been assigned to more than one altitude zone, habitat, or botanical province as appropriate.

\section{RESULTS AND DISCUSSION}

This paper is based on a conservation assessment of the entire indigenous New Zealand vascular plant flora that has identified 792 described taxa of indigenous New Zealand vascular plants (or $34 \%$ of the total indigenous vascular flora) as threatened or nonresident native (Appendix 1). Of these (with percentages of the total indigenous vascular flora given in brackets), 4 (0.2\%) are believed Extinct, 122 (5.3\%) Acutely Threatened, 96 (4.2\%) Chronically Threatened, and a further 525 (23\%) have been assessed as naturally uncommon (At Risk or Non-resident Native). Forty-five candidate taxa have been assessed as Data Deficient because there was insufficient information to provide a more detailed assessment, although there were reasons to believe that they warrant listing. Aside from these listings we have also assessed a further 208 named taxa and unnamed variants (Appendix 2) whose taxonomic status is either unclear, in question, or which have been proposed to the panel as being potentially distinct but which still require further taxonomic assessment and formal recognition. Because the status of these 208 entities is in question we have not included them in the following assessment of the New Zealand Threatened and Uncommon vascular flora. Furthermore, we have not directly compared the current listing with previous threatened plant listings (Cameron et al. 1993, 1995; de Lange et al. 1999)

Table 1. Number of taxonomically determinate threatened and uncommon New Zealand plants with respect to plant family (only families $\geq 10$ taxa are included).

\begin{tabular}{|c|c|c|c|c|c|c|}
\hline Family & Extinct & $\begin{array}{l}\text { Acutely } \\
\text { threatened }\end{array}$ & $\begin{array}{l}\text { Chronically } \\
\text { threatened }\end{array}$ & $\begin{array}{l}\text { Naturally } \\
\text { uncommon }\end{array}$ & $\begin{array}{c}\text { Data } \\
\text { deficient }\end{array}$ & Totals \\
\hline Asteraceae & & 18 & 10 & 101 & 6 & 135 \\
\hline Poaceae & & 9 & 4 & 64 & 66 & 83 \\
\hline Plantaginaceae & & 11 & 5 & 49 & 1 & 66 \\
\hline Cyperaceae & & 4 & 8 & 35 & 4 & 51 \\
\hline Orchidaceae & & 4 & 3 & 23 & 4 & 34 \\
\hline Boraginaceae & & 10 & 2 & 14 & 5 & 31 \\
\hline Apiaceae & & 3 & 1 & 26 & & 30 \\
\hline Ranunculaceae & & 3 & 4 & 16 & & 23 \\
\hline Brassicaceae & 1 & 8 & 6 & 4 & 2 & 21 \\
\hline Fabaceae & & 9 & 5 & 7 & & 21 \\
\hline Ericaceae & & 3 & & 11 & & 14 \\
\hline Onagraceae & & & 1 & 10 & 3 & 14 \\
\hline Araliaceae & & & 2 & 10 & 1 & 13 \\
\hline Gentianaceae & & 1 & & 11 & & 12 \\
\hline Pittosporaceae & & 5 & 1 & 5 & & 11 \\
\hline Rubiaceae & & 2 & 3 & 4 & 1 & 9 \\
\hline Totals & 1 & 90 & 55 & 390 & 32 & 568 \\
\hline
\end{tabular}


because the systems used by those publications to assess candidate threatened or uncommon taxa (IUCN 1994; de Lange \& Norton 1998) differ markedly from the one now used by the Department of Conservation (Molloy et al. 2002). Nevertheless, as with previous listings, some general comment can be made on the nature and extent of threatened and uncommon taxa within the New Zealand vascular flora.

The main contributing families $(\geq 10$ taxa, with number of taxa in brackets) remain similar to those listed by de Lange et al. (1999) with the notable addition of the Ericaceae (14), Onagraceae (14), and Araliaceae (13), and increased dominance of the Cyperaceae (51) (Table 1). The main contributing families (i.e., those with greater than 30 taxa) remain largely the same though with significant increases in the number of taxa within each, e.g., Asteraceae (135), Poaceae (83), Plantaginaceae (66; which has increased its prominence due to the recircumscription of many genera previously included in the Scrophulariaceae (APG II 2003)), Orchidaceae (33), Boraginaceae (31), and the marked increase in the Cyperaceae which in the 1999 assessment contributed 20 taxa and now has 51. However, if those instances of natural rarity (taxa listed as At Risk and Non-resident Native) and those assessed as Data Deficient are removed, overall values decrease. The Asteraceae still contribute the greatest number with
29 taxa, followed by the Plantaginaceae (19), the Poaceae (13), the Cyperaceae and Boraginaceae each with 12, and the Orchidaceae with 7 (Table 1). While these assessments still reflect the overall contribution that these large families make to the New Zealand vascular flora (Wilton \& Breitwieser 2000; P. J. de Lange \& D. A. Norton unpubl. data), the increased dominance of the Poaceae and Cyperaceae at the expense of previous major contributors the Orchidaceae and Brassicaceae reflects our increasing knowledge of these families, the fine tuning the new threat assessment system allows, and the fact that the current assessment is perhaps the first time since initial listings were conducted (Given 1976) that the entire vascular flora has been assessed from a conservation perspective. The same reasons explain why the Ericaceae (including Epacridaceae) and Onagraceae are now so prominent.

Several of the most prominent and species-rich genera in the main contributing families (Table 1) dominate the generic assessment of the lists (Table 2). However, while formerly important genera such as Hebe (45), Myosotis (30), and Celmisia (25) remain dominant, other species-rich yet formerly under-represented genera such as Chionochloa (14) and Epilobium (13) are now significant contributors, whilst Carex (34), Leptinella (18), and Aciphylla (17) have increased their dominance. When these figures are adjusted to include only those threatened

Table 2 Number of taxonomically determinate threatened and uncommon New Zealand plants with respect to plant genus (only genera $\geq 10$ taxa are included).

\begin{tabular}{|c|c|c|c|c|c|c|}
\hline Genus & Extinct & $\begin{array}{l}\text { Acutely } \\
\text { threatened }\end{array}$ & $\begin{array}{l}\text { Chronically } \\
\text { threatened }\end{array}$ & $\begin{array}{l}\text { Naturally } \\
\text { uncommon }\end{array}$ & $\begin{array}{c}\text { Data } \\
\text { deficient }\end{array}$ & Totals \\
\hline Hebe & & 9 & 1 & 35 & 1 & 45 \\
\hline Carex & & 2 & 3 & 25 & 4 & 34 \\
\hline Myosotis & & 9 & 2 & 14 & 5 & 30 \\
\hline Celmisia & & 1 & 1 & 23 & & 25 \\
\hline Leptinella & & 4 & 1 & 13 & & 18 \\
\hline Ranunculus & & 1 & 3 & 14 & & 18 \\
\hline Aciphylla & & 2 & & 15 & & 17 \\
\hline Poa & & 2 & & 14 & & 16 \\
\hline Olearia & & 5 & 2 & 8 & 1 & 16 \\
\hline Senecio & & 2 & & 13 & & 15 \\
\hline Carmichaelia & & 7 & 3 & 4 & & 14 \\
\hline Chionochloa & & & & 14 & & 14 \\
\hline Epilobium & & & 1 & 9 & 3 & 13 \\
\hline Brachyglottis & & 1 & 3 & 7 & & 11 \\
\hline Gentiana & & & & 11 & & 11 \\
\hline Lepidium & 1 & 6 & 2 & 1 & 1 & 11 \\
\hline Pittosporum & & 5 & 1 & 5 & & 11 \\
\hline Totals & 1 & 56 & 23 & 227 & 15 & 322 \\
\hline
\end{tabular}


(Acute and Chronically Threatened), the overall contribution of species-rich genera characterised by highly localised but not necessarily threatened taxa (e.g., Carex and Celmisia) drops, and Myosotis (11), Hebe (10), Carmichaelia (10), and Lepidium (8) become prominent, mirroring patterns in the previous list (de Lange et al. 1999).

With respect to life form, dicotyledonous composite (103) and non-composite herbs (233) comprise $42.4 \%$ of the listings, followed by dicotyledonous trees (52) and shrubs (155) which contribute another 26\% (Table 3). Monocotyledonous contributions are dominated by grasses $(83 ; 10.4 \%)$, sedges $(51 ; 6.5 \%)$, and orchids (33; $4.2 \%)$, while ferns (40) provide $5 \%$ of all listings. These patterns are comparable to those observed by de Lange et al. (1999). Again, if these figures are adjusted to include only those threatened (Acute and Chronically Threatened), non-composite (67) and composite dicotyledonous herbs (17) still make up the majority of threatened taxa (38.8\%), followed by dicotyledonous shrubs (48) and trees (24) which comprise $32.9 \%$ of all threatened plant listings. Notably, the number of threatened grasses drops from 83 taxa to 13 (5.9\% of threatened plant listings), reflecting the high incidence of natural rarity within this group rather than actual threatened status. Indeed, of those grass species listed only Amphibromus fluitans, Cortaderia turbaria,
Puccinellia raroflorens, and Puccinellia walkeri subsp. chathamica are under serious active threat, the majority of the remaining species being listed as threatened merely because they occupy small vulnerable habitats and have low overall population sizes (cf. Molloy et al. 2002). Sedges (12) also have only a few species actively at risk from external threats (e.g., Carex inopinata and C. dolomitica); the remaining threatened species are listed because of overall small population size, vulnerable habitats, or through gradual deterioration in overall habitat quality (e.g., Carex litorosa). There are also far fewer threatened than naturally uncommon orchids (7; $3.2 \%$ ), two of which, Anzybas (Corybas) carsei and Linguella (Pterostylis) puberula, are doubtfully endemic species with very close (if not conspecific) relatives in Australia.

With regard to altitudinal zones (Table 4), most taxa are found within lowland (268), montane (246), and coastal habitats (187), while there is a virtually even split of taxa occupying alpine (142) and subalpine (140) zones, appreciating that some taxa span a number of different altitudinal zones. These figures change markedly when Naturally Uncommon and Data Deficient categories are removed. Though lowland (103), montane (86), and coastal (55) zones still contribute the most threatened taxa, there are far fewer taxa within the alpine (14) and subalpine (20) zones. These patterns reflect the fact

Table 3 Number of taxonomically determinate threatened and uncommon New Zealand plants with respect to life form.

\begin{tabular}{|c|c|c|c|c|c|c|}
\hline Life form & Extinct & $\begin{array}{l}\text { Acutely } \\
\text { threatened }\end{array}$ & $\begin{array}{l}\text { Chronically } \\
\text { threatened }\end{array}$ & $\begin{array}{l}\text { Naturally } \\
\text { uncommon }\end{array}$ & $\begin{array}{c}\text { Data } \\
\text { deficient }\end{array}$ & Totals \\
\hline Non-composite dicot herbs & 2 & 34 & 33 & 142 & 21 & 232 \\
\hline Dicot shrubs & 1 & 30 & 18 & 102 & 4 & 155 \\
\hline Composite dicot herbs & & 12 & 5 & 83 & 3 & 103 \\
\hline Grasses & & 9 & 4 & 64 & 6 & 83 \\
\hline Sedges & & 4 & 8 & 35 & 4 & 51 \\
\hline Dicot trees & & 15 & 9 & 24 & & 48 \\
\hline Ferns & & 5 & 6 & 28 & 1 & 40 \\
\hline Orchids & & 4 & 3 & 23 & 3 & 33 \\
\hline Other monocot herbs & & 3 & 4 & 6 & 2 & 15 \\
\hline Hemiparasites & 1 & & 5 & 5 & 1 & 12 \\
\hline Rushes \& allied plants & & 1 & 1 & 5 & & 7 \\
\hline Dicot lianes & & 2 & & 2 & & 4 \\
\hline Fern allies & & 2 & & 1 & & 3 \\
\hline Conifers & & & & 2 & & 2 \\
\hline Monocot trees & & & & 1 & & 1 \\
\hline Saprophytes & & & & 1 & & 1 \\
\hline Parasites & & & 1 & & & 1 \\
\hline
\end{tabular}


that most taxa within subalpine and alpine habitats are simply narrow-range endemics or sparsely distributed taxa and are not directly threatened (cf. de Lange \& Norton 1998).

In terms of habitat type (Table 5), cliff-dwelling taxa dominate the listings with 206 taxa, 53 of which are Threatened. Perhaps surprisingly, closed forest constitutes the next major contributing habitat type with 154 taxa (59 listed as Threatened), followed by
108 taxa which occupy boulder field, talus, and scree habitats; few of the remaining habitat types contributed more than 50 taxa, and most of these become negligible contributors ( $<10$ taxa) when Naturally Uncommon and Data Deficient groupings are removed. Indeed, only boulder field, talus, and scree (22), turf and cushion (15), beach (21), grey scrub (18), and oligotrophic (10) and eu-mesotrophic (15) wetland habitats contribute $\geq 10$ threatened taxa.

Table 4 Number of taxonomically determinate threatened and uncommon New Zealand plants with respect to altitudinal zone.

\begin{tabular}{lccccrr}
\hline $\begin{array}{l}\text { Altitudinal } \\
\text { zone }\end{array}$ & Extinct & $\begin{array}{c}\text { Acutely } \\
\text { threatened }\end{array}$ & $\begin{array}{c}\text { Chronically } \\
\text { threatened }\end{array}$ & $\begin{array}{c}\text { Naturally } \\
\text { uncommon }\end{array}$ & $\begin{array}{c}\text { Data } \\
\text { deficient }\end{array}$ & Totals \\
\hline Lowland & 1 & 53 & 50 & 148 & 13 & 265 \\
Montane & 1 & 47 & 38 & 137 & 23 & 246 \\
Coastal & 1 & 29 & 27 & 125 & 5 & 187 \\
Alpine & 1 & 5 & 9 & 121 & 7 & 142 \\
Subalpine & & 8 & 12 & 107 & 12 & 140 \\
\hline
\end{tabular}

Table 5 Number of taxonomically determinate threatened and uncommon New Zealand plants with respect to major habitats.

\begin{tabular}{|c|c|c|c|c|c|c|}
\hline Habitat & Extinct & $\begin{array}{l}\text { Acutely } \\
\text { threatened }\end{array}$ & $\begin{array}{l}\text { Chronically } \\
\text { threatened }\end{array}$ & $\begin{array}{l}\text { Naturally } \\
\text { uncommon }\end{array}$ & $\begin{array}{c}\text { Data } \\
\text { deficient }\end{array}$ & Totals \\
\hline Cliff & 1 & 34 & 19 & 146 & 6 & 206 \\
\hline Closed forest & 1 & 32 & 27 & 87 & 7 & 154 \\
\hline $\begin{array}{l}\text { Boulder field, talus, } \\
\text { and scree }\end{array}$ & & 13 & 9 & 80 & 6 & 108 \\
\hline Turf and cushion & 2 & 6 & 9 & 34 & 3 & 54 \\
\hline Fell field and herbfield & & & 2 & 47 & 3 & 52 \\
\hline Beach & 1 & 9 & 12 & 29 & 1 & 52 \\
\hline Flush and seepages & & 5 & 2 & 28 & 7 & 42 \\
\hline Tall-tussock grassland & & 1 & 1 & 36 & 4 & 42 \\
\hline Coastal scrub & & 6 & 1 & 29 & 1 & 37 \\
\hline Short-tussock grassland & & 3 & 5 & 22 & 5 & 35 \\
\hline Oligotrophic wetland & & 6 & 4 & 23 & & 33 \\
\hline Grey scrub & & 10 & 8 & 9 & 1 & 28 \\
\hline Eu-/mesotrophic wetland & & 6 & 9 & 9 & 2 & 26 \\
\hline Other scrub & & 4 & 3 & 18 & & 25 \\
\hline Subalpine scrub & & 2 & & 16 & & 18 \\
\hline Estuary & & 2 & 3 & 11 & & 16 \\
\hline River bed & & 3 & 3 & 6 & 3 & 15 \\
\hline Aquatic & & & 6 & 3 & 1 & 10 \\
\hline Geothermal & & & 2 & 5 & & 7 \\
\hline Inland saline & & 3 & & 1 & 1 & 5 \\
\hline Open forest & & 1 & & 2 & & 3 \\
\hline Other grassland & & & 1 & 1 & & 2 \\
\hline Wetland margin & & & & & & \\
\hline
\end{tabular}


Table 6 Number of taxonomically determinate threatened and uncommon New Zealand plants with respect to botanical provinces (after Wardle 1991).

\begin{tabular}{|c|c|c|c|c|c|c|}
\hline $\begin{array}{l}\text { Botanical } \\
\text { province }\end{array}$ & Extinct & $\begin{array}{l}\text { Acutely } \\
\text { threatened }\end{array}$ & $\begin{array}{l}\text { Chronically } \\
\text { threatened }\end{array}$ & $\begin{array}{l}\text { Naturally } \\
\text { uncommon }\end{array}$ & $\begin{array}{c}\text { Data } \\
\text { deficient }\end{array}$ & Totals \\
\hline Otago & 1 & 28 & 45 & 105 & 19 & 198 \\
\hline Canterbury & 1 & 39 & 48 & 84 & 19 & 191 \\
\hline Northland & 1 & 35 & 38 & 84 & 5 & 163 \\
\hline Auckland & 1 & 28 & 49 & 66 & 7 & 151 \\
\hline Western Nelson & & 21 & 34 & 81 & 13 & 149 \\
\hline Southern North I. & 2 & 24 & 48 & 56 & 12 & 142 \\
\hline Marlborough & & 14 & 36 & 67 & 16 & 133 \\
\hline Southland & 1 & 9 & 40 & 70 & 7 & 127 \\
\hline Volcanic Plateau & 1 & 11 & 35 & 44 & 10 & 101 \\
\hline Sounds-Nelson & & 9 & 19 & 46 & 6 & 80 \\
\hline Rakiura & & 4 & 17 & 54 & 5 & 80 \\
\hline Gisborne & & 9 & 31 & 28 & 8 & 76 \\
\hline Taranaki & & 8 & 26 & 27 & 6 & 65 \\
\hline Westland & & 5 & 19 & 36 & 6 & 66 \\
\hline Fiordland & & 2 & 18 & 42 & 5 & 67 \\
\hline Chatham & & 16 & 12 & 36 & 2 & 66 \\
\hline Campbell & & 1 & & 55 & 3 & 59 \\
\hline Three Kings & & 11 & 6 & 21 & 1 & 39 \\
\hline Kermadec & & 5 & 2 & 29 & & 36 \\
\hline
\end{tabular}

When compared with de Lange et al. (1999), the distribution of threatened and uncommon taxa by botanical province within New Zealand (Table 6) has seen the elevation of Otago (198) and Canterbury (191) above such traditional species-rich "hot spots" as Northland (163) and Western Nelson (149). Notable also has been the increased prominence of Auckland (151) and the southern North Island (142). These differences reflect the use of the Molloy et al. (2002) threat classification scheme and the much larger data base sampled. Again, a more meaningful impression is derived through removal of Naturally Uncommon and Data Deficient categories. In this situation it is Canterbury (87), followed by Auckland (77), Otago (73), Northland (73), and the southern North Island (72) which contain the largest numbers of threatened taxa.

\section{ACKNOWLEDGMENTS}

This paper has benefited from the specialist advice received from the following people: M. Bayly (Plantaginaceae), F. Venter (Dracophyllum), D. Glenny (Aciphylla, Gentiana), B. Parris (Doodia), K. Lloyd (Chionochloa, Festuca), H. E. Connor (Poaceae), E. Edgar (Poaceae), P. J. Brownsey (Pteridophyta), P. J.
Garnock-Jones (Brassicaceae, Plantaginaceae, Ranunculaceae), L. Perrie (Polystichum), D. Cameron (Victorian congenerics (Australia)), H. R. Toelken (Crassula, Kunzea), H. D. Wilson (Banks Peninsula endemic plants), G. Walls (Chatham Island endemic plants), A. Wilton (Luzula), I. St George (Orchidaceae), M. Chase (Angiosperm families and genera), and E. Scanlen (Orchidaceae). We also thank the New Zealand Department of Conservation botanists S. Dopson, L. J. Forester, R. Stanley, J. Boow, A. Brandon, P. Cashmore, M. Thorsen, G. La Cock, N. Singers, J. W. D. Sawyer, T. Silbery, C. Jones, N. Head, J. Barkla, and P. Knightbridge for sharing their extensive knowledge of the New Zealand indigenous flora, their advice, and critique of earlier drafts of this paper.

\section{REFERENCES}

APG II 2003: An update of the Angiosperm Phylogeny Group classification for the orders and families of flowering plants: APG II. Botanical Journal of the Linnean Society 141: 399-436.

Brummitt, R. K.; Powell, C. E. 1992: Authors of plant names. Kew, Royal Botanic Gardens.

Cameron, E. K.; de Lange, P. J.; Given, D. R.; Johnson, P. N.; Ogle, C. C. 1993: New Zealand Botanical Society threatened and local plant lists (1993 revision). New Zealand Botanical Society Newsletter 32: 14-28. 
Cameron, E. K.; de Lange, P. J.; Given, D. R.; Johnson, P. N.; Ogle, C. C. 1995: New Zealand Botanical Society threatened and local plant lists (1995 revision). New Zealand Botanical Society Newsletter 39: 15-28.

Cheeseman, T. F. 1901: Some recent additions to the New Zealand flora. Transactions of the New Zealand Institute 33: 312-313.

de Lange, P. J.; Murray, B. G. 2002: Contributions to a chromosome atlas of the New Zealand flora -37 . Miscellaneous families. New Zealand Journal of Botany 40: 1-23.

de Lange, P. J.; Norton, D. A. 1998: Revisiting rarity: a botanical perspective on the meanings of rarity and the classification of New Zealand's uncommon plants. In: Lynch, R. ed. Ecosystems, entomology and plants. The Royal Society of New Zealand Miscellaneous Series 48: 145-160.

de Lange, P. J.; Heenan, P. B.; Given, D. R.; Norton, D. A.; Ogle, C. C.; Johnson, P. N.; Cameron, E. K. 1999: Threatened and uncommon plants of New Zealand. New Zealand Journal of Botany 37: 603-628.

Given, D. R. 1976: A register of rare and endangered indigenous plants in New Zealand. New Zealand Journal of Botany 14: 135-149.

Hitchmough, R. 2001: Threatened plant list. New Zealand Botanical Society Newsletter 65: 19-20.

Hitchmough, R. 2002: New Zealand threat classification system lists. Wellington, Department of Conservation.
IUCN 1994: IUCN red list categories. Gland, Switzerland, IUCN Species Survival Commission.

IUCN 2000: IUCN red list categories. Gland, Switzerland, IUCN Species Survival Commission.

Kubitzki, K. ed. 1990: The families and genera of vascular plants. Vol. I. Pteridophytes and gymnosperms. Berlin, Springer-Verlag.

Molloy, J.; Davis, A. 1992: Setting priorities for the conservation of New Zealand's threatened plants and animals. Wellington, Department of Conservation.

Molloy, J.; Bell, B.; Clout, M.; de Lange, P.; Gibbs, G.; Given, D.; Norton, D.; Smith, N.; Stephens, T. 2002: Classifying species according to threat of extinction - a system for New Zealand. Wellington, Department of Conservation.

Moore, L. B.; Edgar, E. 1970: Flora of New Zealand. Vol. II. Wellington, Government Printer.

Rogan, D. 2002: New Zealand Botanical Society Annual General Meeting minutes. New Zealand Botanical Society Newsletter 67: 2-4.

Scanlen, E. 2003: The Column. 2. Chiloglottis round up. The New Zealand Native Orchid Journal 86: 14-15.

Wardle, P. 1991: The vegetation of New Zealand. Cambridge, Cambridge University Press.

Wilton, A. Breitwieser, I. 2000: Composition of the New Zealand seed plant flora. New Zealand Journal of Botany 38: 537-549. 
Appendix 1 New Zealand threatened and uncommon vascular plant list.

$\dagger$ denotes indigenous taxa found naturally outside New Zealand.

$\$$ denotes an addition to this list (cf. de Lange et al. 1999).

\section{Qualifiers}

With the exception of Partial Decline (PD) and Island Endemic (IE) full definitions are provided for the qualifiers used in this list by Molloy et al. (2002). Partial Decline has been used here to indicate taxa (e.g., Hebe acutiflora) whose populations are subject to range contraction below the minimum thresholds used by Molloy et al. (2002) for Gradual Decline. Island Endemic refers to taxa (e.g., Corokia macrocarpa) confined to a single archipelago beyond the three main islands of New Zealand.

EW Extinct in the Wild

CD Conservation Dependent

DP Data Poor

RC Recovering

ST Stable

SO Secure Overseas

TO Threatened Overseas

HI Human Induced

RF Recruitment Failure

EF Extreme Fluctuations

OL One Location

PD Partial Decline

IE Island Endemic

\section{Extinct (4)}

A taxon is listed as Extinct when there is no reasonable doubt, after repeated surveys in known or expected habitats at appropriate times (diurnal, seasonal, and annual) and throughout the taxon's historic range, that the last naturally occurring individual has died. Only taxa which have become extinct since 1840 are included in this list.

Lepidium obtusatum Kirk

Logania depressa Hook.f.

Stellaria elatinoides Hook.f.

Trilepidea adamsii (Cheeseman) Tiegh.
Brassicaceae

Loganiaceae

Caryophyllaceae

Loranthaceae

\section{Acutely Threatened (122)}

Acutely Threatened taxa are those which meet the criteria specified by Molloy et al. (2002) for the categories 1 . Nationally Critical, 2. Nationally Endangered, and 3. Nationally Vulnerable.

\section{Nationally Critical (47)}

Listed here are those taxa which qualify as Nationally Critical, because of their small population size ( $\leq 250$ mature individuals), or the number of sub-populations known ( $\leq 2$, with $\leq 200$ mature individuals in the largest of these), or their area of occupancy $\left(0.01 \mathrm{~km}^{2}\right)$, or their predicted decline rate $(\geq 80 \%$ in the next 10 years). Fuller definitions are provided by Molloy et al. (2002).

Acaena rorida B.H.Macmill. $\mathrm{CD}$, OL

Alectryon excelsus subsp. grandis (Cheeseman) de Lange et E.K.Cameron $\mathrm{RC}$, IE

Anzybas carsei (Cheeseman) D.L.Jones et M.A.Clem. CD, HI, RF, EF, OL

Atriplex hollowayi de Lange et D.A.Norton $\mathrm{CD}, \mathrm{EF}$

Brachyscome pinnata Hook.f. $\mathrm{CD}$, OL

Carex dolomitica Heenan et de Lange $\mathrm{CD}, \mathrm{OL}$

Carmichaelia hollowayi G.Simpson $\mathrm{CD}, \mathrm{RF}$

C. muritai (A.W.Purdie) Heenan ${ }_{\mathrm{CD}}$

Celmisia macmahonii Kirk var. macmahonii ${ }_{\mathrm{ST}}$

${ }^{\dagger}$ Centipeda minima (L.) A.Braun et Asch. subsp. minima SO, EF

Ceratocephala pungens Garn.-Jones EF, HI

${ }^{\dagger}$ Christella dentata (Forssk.) Brownsey et Jermy sens. str. CD, RF, OL, SO, HI

Clianthus puniceus (G.Don) Sol. ex Lindl. CD, OL, HI

Cortaderia turbaria Connor $\mathrm{CD}$, RF, IE

Coprosma spathulata subsp. hikuruana de Lange et Heenan $\mathrm{CD}, \mathrm{HI}, \mathrm{OL}$

Crassula hunua A.P.Druce ${ }_{\mathrm{HI}}$

Davallia tasmanii subsp. cristata von Konrat, Braggins et de Lange $\mathrm{CD}$, ST, RF, OL

Gunnera hamiltonii Kirk CD, ST, RF
Rosaceae

Sapindaceae

Orchidaceae

Amaranthaceae

Asteraceae

Cyperaceae

Fabaceae

Fabaceae

Asteraceae

Asteraceae

Ranunculaceae

Thelypteridaceae

Fabaceae

Poaceae

Rubiaceae

Crassulaceae

Davalliaceae

Gunneraceae 
Hebe breviracemosa (W.R.B.Oliv.) Cockayne et Allan CD, HI, OL, IE

$\ddagger$ H. societatis Bayly et Kellow DP, OL

Lepidium banksii Kirk $\mathrm{CD}$, HI

L. sisymbrioides subsp. matau (Petrie) Thell. CD, HI, EF

Leptinella filiformis (Hook.f.) D.G.Lloyd et C.Webb EW, CD, HI

Linguella puberula Hook.f. HI, EF

Mazus novaezeelandiae subsp. impolitus f. hirta Heenan $\mathrm{CD}, \mathrm{HI}$

Metrosideros bartlettii J.W.Dawson $\mathrm{CD}$, HI

Myosotis albosericea Hook.f. ST, OL

M. angustata Cheeseman $\mathrm{ST}, \mathrm{OL}$

M. australis var. lytteltonensis Laing et $\mathrm{A}$.Wall $\mathrm{CD}, \mathrm{HI}, \mathrm{EF}$

M. cheesemanii Petrie $\mathrm{DP}, \mathrm{EF}$

M. petiolata Hook.f. var. petiolata $\mathrm{DP}, \mathrm{HI}, \mathrm{OL}$

Neopaxia drucei Heenan ST, OL

Olearia gardneri Heads $\mathrm{CD}, \mathrm{HI}, \mathrm{RF}$

Pachycladon exilis (Heenan) Heenan et A.Mitch. CD, HI, OL

Pennantia baylisiana (W.R.B.Oliv.) G.T.S.Baylis CD, ST, RF, OL, IE

Poa spania Edgar et Molloy $\mathrm{CD}, \mathrm{OL}$

P. sudicola Edgar DP, ST

$\dagger$ Pomaderris apetala subsp. maritima N.G.Walsh et F.Coates $\mathrm{CD}, \mathrm{SO}, \mathrm{RF}$

Pterostylis micromega Hook.f. $\mathrm{CD}, \mathrm{HI}, \mathrm{EF}$

Puccinellia raroflorens Edgar $\mathrm{CD}$, DP

$¥ P$. walkeri subsp. chathamica (Cheeseman) Edgar DP

†Sebaea ovata (Labill.) R.Br. CD, SO, HI, EF

\$Sicyos australis Endl. CD, TO

Tecomanthe speciosa W.R.B.Oliv. CD, RF, OL, IE

Thelymitra sanscilia Hatch $\mathrm{DP}, \mathrm{EF}$

$\$$ Uncinia perplexa Heenan et de Lange $\mathrm{CD}$, HI, OL

Wahlenbergia pygmaea subsp. tararua J.A.Petterson DP, ST

Plantaginaceae

Plantaginaceae

Brassicaceae

Brassicaceae

Asteraceae

Orchidaceae

Phrymaceae

Myrtaceae

Boraginaceae

Boraginaceae

Boraginaceae

Boraginaceae

Boraginaceae

Portulacaceae

Asteraceae

Brassicaceae

Pennantiaceae

Poaceae

Poaceae

Rhamnaceae

Orchidaceae

Poaceae

Poaceae

Gentianaceae

Cucurbitaceae

Bignoniaceae

Orchidaceae

Cyperaceae

Campanulaceae

\section{Nationally Endangered (54)}

Listed here are those taxa characterised by their small population size $(250-1000$ mature individuals), $\leq 5$ subpopulations known (with either $\leq 300$ mature individuals in the largest population or the total area of occupancy $\leq 0.1 \mathrm{~km}^{2}$ ), and a moderate to high recent predicted decline ( $\geq 30 \%$ of the total population or habitat area over the last 100 years, or predicted to occur within the next 10 years); or those taxa typified by small to moderate population sizes (1000-5000 mature individuals), $\leq 15$ sub-populations (with $\leq 200-500$ mature individuals in the largest or the total area of occupancy is $\left.0.1-1 \mathrm{~km}^{2}\right)$, and a high recent or predicted decline ( $\geq 60 \%$ of the total population or habitat area over the last 100 years, or this is predicted to occur within the next 10 years). Fuller definitions are provided by Molloy et al. (2002).

Aciphylla traversii (F.Muell.) Hook.f. CD, EF, OL

$\ddagger$ Ackama nubicola de Lange $\mathrm{CD}, \mathrm{RF}, \mathrm{OL}$

${ }^{\dagger}$ Amphibromus fluitans Kirk $\mathrm{EF}, \mathrm{TO}$

Asplenium pauperequitum Brownsey et P.Jackson CD, EF, HI, OL, IE

Astelia chathamica (Skottsb.) L.B.Moore $\mathrm{CD}, \mathrm{EF}, \mathrm{IE}$

Australopyrum calcis Connor et Molloy subsp. calcis $\mathrm{CD}$, ST, OL

Boehmeria australis var. dealbata (Cheeseman) Sykes OL, IE

Brachyglottis huntii (F.Muell.) B.Nord. CD, RC, HI, IE

${ }^{\ddagger}$ Bulbinella modesta L.B.Moore ${ }_{\text {DP }}$

Carex inopinata Cook $\mathrm{CD}$

Carmichaelia curta Petrie ${ }_{\mathrm{HI}}$

C. juncea Hook.f. CD, EF, HI

C. kirkii Hook.f. DP

C. williamsii Kirk $\mathrm{HI}$

Clianthus maximus Colenso

Coprosma waima A.P.Druce $\mathrm{CD}, \mathrm{RC}$

Cortaderia turbaria Connor $\mathrm{CD}, \mathrm{RF}, \mathrm{IE}$

${ }^{\dagger}$ Crassula peduncularis (Smith) F.Meigen $\mathrm{SO}, \mathrm{EF}$

Embergeria grandifolia (Kirk) Boulos $\mathrm{CD}$, IE

Epacris sinclairii Hook.f. RC

Hebe armstrongii (J.B.Armstr.) Cockayne et Allan CD, HI

H. salicornioides (Hook.f.) Cockayne et Allan $\mathrm{RF}$
Apiaceae

Cunoniaceae

Poaceae

Aspleniaceae

Asteliaceae

Poaceae

Urticaceae

Asteraceae

Asparagaceae

Cyperaceae

Fabaceae

Fabaceae

Fabaceae

Fabaceae

Fabaceae

Rubiaceae

Poaceae

Crassulaceae

Asteraceae

Ericaceae

Plantaginaceae

Plantaginaceae 
H. speciosa (A.Cunn.) Cockayne et Allan $\mathrm{CD}$, HI, RF

Helichrysum dimorphum Cockayne $\mathrm{CD}, \mathrm{HI}, \mathrm{RF}$

Heliohebe raoulii subsp. maccaskillii (Allan) Garn.-Jones

$\dagger$ Juncus holoschoenus R.Br. var. holoschoenus DP, SO

Lepidium kirkii Petrie $\mathrm{CD}$, HI, EF

L. oleraceum Sparrman sens. str. $\mathrm{CD}, \mathrm{HI}, \mathrm{EF}$

L. sisymbrioides subsp. kawarau (Petrie) Thell. CD. HI

Leptinella nana (D.G.Lloyd) D.G.Lloyd et C.Webb $\mathrm{CD}, \mathrm{EF}$

Myosotidium hortensia (Decne.) Baill. CD, IE

Myosotis colensoi (Kirk) Macbride $\mathrm{CD}, \mathrm{EF}$

M. petiolata var. pansa L.B.Moore $\mathrm{EF}$

M. pygmaea var. glauca G.Simpson et J.S.Thomson $\mathrm{CD}$, EF

Myosurus minimus subsp. novae-zelandiae (W.R.B.Oliv.) Garn.-Jones HI, EF

Myrsine argentea Heenan et de Lange $\mathrm{OL}$

Olearia crebra E.K.Cameron et Heenan $\mathrm{CD}$, DP, RC

O. pachyphylla Cheeseman ${ }_{\mathrm{HI}}$

O. polita H.D.Wilson et Garn.-Jones $\mathrm{CD}$

Ophioglossum petiolatum Hook. CD, HI, SO

Oreomyrrhis colensoi var. delicatula Allan CD, DP

$\dagger$ Phylloglossum drummondii Kunze $\mathrm{SO}, \mathrm{HI}, \mathrm{EF}$

$\dagger$ Picris burbidgei S.Holzapfel so

Pittosporum serpentinum (de Lange) de Lange $\mathrm{CD}, \mathrm{RF}, \mathrm{OL}$

P. obcordatum Raoul $\mathrm{CD}, \mathrm{RF}$

P. patulum Hook.f. CD, RF

P. turneri Petrie $\mathrm{CD}, \mathrm{RC}$

$\dagger$ Pomaderris phylicifolia Lodd. so, HI

Rorippa divaricata (Hook.f.) Garn.-Jones et Jonsell CD, EF

Senecio kermadecensis Belcher ${ }_{\mathrm{EF}} \mathrm{IE}$

S. scaberulus (Hook.f.) D.G.Drury HI, EF

Simplicia laxa Kirk $\mathrm{CD}, \mathrm{HI}$

${ }^{\dagger}$ Todea barbara (L.) Moore so

${ }^{\dagger}$ Triglochin palustris $\mathrm{L}$. so

Uncinia strictissima Petrie $\mathrm{DP}, \mathrm{HI}$
Plantaginaceae

Asteraceae

Plantaginaceae

Juncaceae

Brassicaceae

Brassicaceae

Brassicaceae

Asteraceae

Boraginaceae

Boraginaceae

Boraginaceae

Boraginaceae

Ranunculaceae

Myrsinaceae

Asteraceae

Asteraceae

Asteraceae

Ophioglossaceae

Apiaceae

Lycopodiaceae

Asteraceae

Pittosporaceae

Pittosporaceae

Pittosporaceae

Pittosporaceae

Rhamnaceae

Brassicaceae

Asteraceae

Asteraceae

Poaceae

Osmundaceae

Juncaginaceae

Cyperaceae

\section{Nationally Vulnerable (21)}

Listed here are those taxa characterised by their small to moderate population size (1000-5000 mature individuals), $\leq 15$ sub-populations (either with 300-500 mature individuals in the largest sub-population or occupying a total area of $0.1-1 \mathrm{~km}^{2}$ ), and with either an initially historic but continuing decline rate of 30-60\% in total population size or habitat area over the last 100 years, or a predicted decline of $30-60 \%$ in the total population likely in the next 10 years. Fuller definitions are provided by Molloy et al. (2002).

Aciphylla dieffenbachii Kirk $\mathrm{CD}$, EF, IE

Australopyrum calcis subsp. optatum Connor et Molloy $\mathrm{CD}$

Carmichaelia carmichaeliae (Hook.f.) Heenan

$\$$ Dracophyllum longifolium var. septentrionale W.R.B.Oliv. RF, OL

$\$$ D. urvilleanum A.Rich. DP

Hebe barkeri (Cockyane) Cockayne $\mathrm{CD}$, OL

H. bishopiana (Petrie) Hatch ${ }_{\mathrm{CD}}$

H. cupressoides (Hook.f.) Cockayne et Allan $\mathrm{CD}, \mathrm{HI}$

H. perbella de Lange

H. scopulorum Bayly, de Lange et Garn.-Jones CD

${ }^{\dagger}$ Hibiscus diversifolius Jacq. so

Lepidium flexicaule Kirk $\mathrm{CD}$, SO, EF

Leptinella featherstonii F.Muell. $\mathrm{CD}$, IE

L. rotundata (Cheeseman) D.G.Lloyd et C.Webb

$\dagger$ Lycopodiella serpentina (Kunze) B.Øllg. TO

Muehlenbeckia astonii Petrie ${ }_{\mathrm{CD}} \mathrm{RF}$

${ }^{\ddagger}$ Myosotis pygmaea var. minutiflora G.Simpson et J.S.Thomson $\mathrm{CD}$, EF

Olearia hectorii Hook.f. $\mathrm{CD}$, RF

Pittosporum dallii Cheeseman $\mathrm{CD}, \mathrm{RF}$

Ranunculus ternatifolius Kirk

Scutellaria novae-zelandiae Hook.f. $\mathrm{CD}$
Apiaceae

Poaceae

Fabaceae

Ericaceae

Ericaceae

Plantaginaceae

Plantaginaceae

Plantaginaceae

Plantaginaceae

Plantaginaceae

Malvaceae

Brassicaceae

Asteraceae

Asteraceae

Lycopodiaceae

Polygonaceae

Boraginaceae

Asteraceae

Pittosporaceae

Ranunculaceae

Lamiaceae 
Chronically Threatened (97)

Chronically Threatened taxa are those which meet the criteria specified by Molloy et al (2002) for the categories 1. Serious Decline and 2. Gradual Decline.

\section{Serious Decline (26)}

Taxa qualify if they occur as moderate to large populations where there is a moderate to large predicted decline (with total population size $>5000$ mature individuals, $>15$ sub-populations and either $>500$ mature individuals in the largest sub-population or the total area of occupancy $>1 \mathrm{~km}^{2}$, with a predicted decline rate of $>30 \%$ in total population over the next 10 years), or taxa exist as small to moderate sized populations with a small to moderate predicted decline (with total population $<5000$ mature individuals, $\leq 500$ mature individuals in the largest subpopulation or total area of occupancy $\leq 1 \mathrm{~km}^{2}$, with a predicted decline rate of 5-30\% in the total population over the next 10 years). Fuller definitions are provided by Molloy et al. (2002).

${ }_{B}$ Brachyglottis kirkii (Kirk) C.Webb var. kirkii CD, DP

Carex litorosa Bailey DP, HI

Carmichaelia vexillata Heenan $\mathrm{RF}$

Dactylanthus taylorii Hook.f. CD, RF

$\$$ Daucus glochidiatus (Labill.) Fisch., C.A.Mey. \& Avé-Lall. SO, DP

Drymoanthus flavus St George et Molloy

Euphorbia glauca G.Forst. EF

Heliohebe acuta Garn.-Jones CD, DP

${ }^{\ddagger}$. lavaudiana (Raoul) Garn.-Jones $\mathrm{CD}$

Hydatella inconspicua (Cheeseman) Cheeseman $\mathrm{EF}$

Isolepis basilaris Hook.f. HI, EF

${ }^{\dagger}$ Kunzea ericoides var. linearis (Kirk) W.Harris $\mathrm{HI}$

Leucogenes tarahaoa Molloy oL

Luzula celata Edgar

$\dagger$ Marattia salicina Smith $\mathrm{CD}$, SO

Mazus novaezeelandiae subsp. impolitus Heenan f. impolitus $\mathrm{CD}$, HI

M. novaezeelandiae W.R.Barker subsp. novaezeelandiae $\mathrm{CD}, \mathrm{HI}$

Myosotis pygmaea Colenso var. pygmaea $\mathrm{CD}$, DP

Olearia fimbriata Heads

Pachycladon stellata (Allan) Heenan et A.Mitch. DP

Pimelea tomentosa (J.R.Forst. et G.Forst.) Druce sens. str. EF

$\ddagger$ Pittosporum kirkii Hook.f. CD, DP

Plagianthus chathamicus Cockayne $\mathrm{CD}$, RC, IE

$\dagger$ Plumatochilos tasmanicum (D.L.Jones) D.L.Szlachetko so, HI, EF

Pterostylis paludosa D.L.Jones, Molloy et M.A.Clem EF

Tetrachondra hamiltonii D.Oliver $\mathrm{DP}$
Asteraceae
Cyperaceae
Fabaceae
Balanophoraceae
Apiaceae
Orchidaceae
Euphorbiaceae
Plantaginaceae
Plantaginaceae
Hydatellaceae
Cyperaceae
Myrtaceae
Asteraceae
Juncaceae
Marattiaceae
Phrymaceae
Phrymaceae
Boraginaceae
Asteraceae
Brassicaceae
Thymelaeaceae
Pittosporaceae
Malvaceae
Orchidaceae
Orchidaceae
Tetrachondraceae

\section{Gradual Decline (70)}

Taxa qualify if they occur as moderate to large populations with small to moderate predicted declines (total population size $>5000$ mature individuals, $>15$ sub-populations and either $>500$ mature individuals in the largest sub-population or the total area of occupancy $>1 \mathrm{~km}^{2}$, with a decline rate of 5-30\% in total population over the next 10 years, which is predicted to continue beyond 10 years). Fuller definitions are provided by Molloy et al. (2002).

$¥$ Acaena buchananii Hook.f. DP

Alepis flavida (Hook.f.) Tiegh. CD, HI

$\dagger$ Anogramma leptophylla (L.) Link SO, EF

${ }^{\dagger}$ Austrofestuca littoralis (Labill.) E.B.Alexev. CD, SO, HI

Brachyglottis perdicioides (Hook.f.) B.Nord. CD

B. sciadophila (Raoul) B.Nord.

¿Carex astonii Hamlin DP

C. cirrhosa Bergg. DP, HI

Carmichaelia crassicaule Hook.f. $\mathrm{RF}$

C. stevensonii (Cheeseman) Heenan

$\ddagger_{\text {Celmisia major Cheeseman var. major }} \mathrm{HI}$

Clematis marmoraria Sneddon $\mathrm{CD}$, RF

Colensoa physaloides (A.Cunn.) Hook.f.

Coprosma obconica Kirk $\mathrm{CD}, \mathrm{RF}$

C. pedicellata Molloy, de Lange et B.D.Clarkson ${ }_{\mathrm{CD}}$

C. wallii Petrie $\mathrm{CD}, \mathrm{RF}$

Corokia macrocarpa Kirk CD, IE

${ }^{\ddagger}$ Crassula kirkii (Allan) A.P.Druce et D.R.Given DP
Rosaceae

Loranthaceae

Pteridaceae

Poaceae

Asteraceae

Asteraceae

Cyperaceae

Cyperaceae

Fabaceae

Fabaceae

Asteraceae

Ranunculaceae

Lobeliaceae

Rubiaceae

Rubiaceae

Rubiaceae

Cornaceae

Crassulaceae 
Crassula manaia A.P.Druce et Sykes $\mathrm{CD}$

$\dagger$ Cyclosorus interruptus (Willd.) H.Itô so

$\dagger$ Deschampsia cespitosa (L.) Beauv. SO. HI

Desmoschoenus spiralis (A.Rich.) Hook.f. CD, EF

$¥$ Doodia squarrosa Colenso

†Drosera pygmaea DC. so

Eleocharis neozelandica Kirk $\mathrm{EF}$

$\$$ Epilobium chionanthum Hauss. DP

Gratiola nana Benth. SO, HI

$\$$ Gunnera arenaria Cheeseman

$¥$ Hebe pimeleoides subsp. faucicola Kellow et Bayly

Iphigenia novae-zelandiae (Hook.f.) Baker ${ }_{\mathrm{DP}} \mathrm{HI}$

${ }^{\dagger}$ Isolepis fluitans (L.) R.Br. SO, HI

$¥$ Jovellana sinclairii (Hook.f.) Kranzl. DP

Lepidium sisymbrioides Hook.f. subsp. sisymbrioides $\mathrm{CD}$

Lepidium tenuicaule Kirk $\mathrm{HI}$

$¥$ Leptinella serrulata (D.G.Lloyd) D.G.Lloyd et C.Webb

Libertia peregrinans Cockayne et Allan CD. HI

Mazus arenarius Heenan, P.N.Johnson et C.Webb

Melicytus flexuosus Molloy et A.P.Druce ${ }_{\mathrm{RF}}$

$\$$ Mida salicifolia A.Cunn. RF

Montigena novae-zelandiae (Hook.f.) Heenan DP, RF

Myosotis brockiei L.B.Moore et M.J.A.Simpson $\mathrm{CD}$

Myriophyllum robustum Hook.f. $\mathrm{CD}$

¥Olearia cheesemanii Cockayne et Allan DP

Ourisia modesta Diels DP

Pachycladon cheesemanii Heenan et A.Mitch. DP

$P$. enysii (Cheeseman) Heenan et A.Mitch.

$P$. fastigiata (Hook.f.) Heenan et A.Mitch.

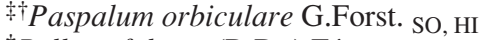

$\dagger$ Pellaea falcata (R.Br.) Fée so, HI

Peraxilla colensoi (Hook.f.) Tiegh. CD, HI

P. tetrapetala Tiegh. $\mathrm{CD}, \mathrm{HI}$

Pimelea arenaria A.Cunn. sens. str. $\mathrm{HI}, \mathrm{RF}$

$\ddagger P$. lyallii Hook.f.

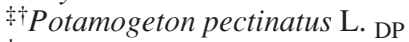

$\ddagger$ Pseudopanax laetus (Kirk) Philipson $\mathrm{RF}$

$\$$ Ranunculus limosella Kirk $\mathrm{HI}$

R. macropus Hook.f. HI

$R$. recens Kirk var. recens $\mathrm{CD}$

$¥$ Raoulia monroi Hook.f ${ }_{\mathrm{EF}}$

$\ddagger$ Raukaua edgerleyi (Hook.f.) Seem. CD, RF

Schoenus carsei Cheeseman ${ }_{\mathrm{HI}}$, SO

Selliera rotundifolia Heenan $\mathrm{CD}$

Sonchus kirkii Hamlin HI, EF

¥Sophora fulvida (Allan) Heenan et de Lange

Teucridium parvifolium Hook.f. $\mathrm{CD}$

Thelypteris confluens (Thunb.) C.Morton $\mathrm{CD}$, SO

${ }^{\ddagger}$ Trisetum antarcticum (G.Forst.) Trin. HI

Tupeia antarctica (G.Forst.) Cham. et Schlecht $\mathrm{CD}$, HI

Urtica linearifolia (Hook.f.) Cockayne ${ }_{\mathrm{CD}}$

$\dagger$ Utricularia australis R.Br. HI

U. delicatula Cheeseman ${ }_{\mathrm{HI}}$
Crassulaceae

Thelypteridaceae

Poaceae

Cyperaceae

Blechnaceae

Droseraceae

Cyperaceae

Onagraceae

Plantaginaceae

Gunneraceae

Plantaginaceae

Colchiaceae

Cyperaceae

Calceolariaceae

Brassicaceae

Brassicaceae

Asteraceae

Iridaceae

Phrymaceae

Violaceae

Santalaceae

Fabaceae

Boraginaceae

Haloragaceae

Asteraceae

Plantaginaceae

Brassicaceae

Brassicaceae

Brassicaceae

Poaceae

Pteridaceae

Loranthaceae

Loranthaceae

Thymelaeaceae

Thymelaeaceae

Potamogetonaceae

Araliaceae

Ranunculaceae

Ranunculaceae

Ranunculaceae

Asteraceae

Araliaceae

Cyperaceae

Goodeniaceae

Asteraceae

Fabaceae

Verbenaceae

Thelypteridaceae

Poaceae

Loranthaceae

Urticaceae

Lentibulariaceae

Lentibulariaceae

\section{At Risk (501)}

These are taxa which do not qualify as Acutely or Chronically Threatened but which exist as widely scattered, small sub-populations or have restricted ranges. Although such taxa are not currently considered threatened, their small population size and aspects of their biology and ecology place them at potential risk, which is why they are listed here as either 1. Sparse or 2. Range Restricted. 


\section{Sparse (126)}

Taxa that, for largely undetermined reasons, occur within typically small and widely scattered populations. This distribution appears wholly natural, and is not considered the result of past or recent anthropogenic disturbance. However, as the candidate taxa usually occur in small numbers at any given site, they are naturally susceptible to extirpation within parts of their range.

$\$$ Aciphylla multisecta Cheeseman

$¥$ A. subflabellata W.R.B.Oliv. DP

Adelopetalum tuberculatum (Colenso) D.L.Jones, M.A.Clem. et Molloy

$¥$ Agrostis imbecilla Zotov

$¥$ A. oresbia Edgar

Anemanthele lessoniana (Steud.) Veldkamp DP

$¥$ Anemone tenuicaulis (Cheeseman) Parkin et Sledge

Anzybas rotundifolius (Hook.f.) D.L.Jones et M.A.Clem.

Asplenium cimmeriorum Brownsey et de Lange

$¥$ A. scleroprium Hombr.

$¥$ Atriplex buchananii (Kirk) Cheeseman

${ }^{\dagger}$ Blechnum norfolkianum (Heward) C.Chr. TO

$\${ }^{\dagger}$ Botrychium australe R.Br. DP, SO, EF

Calochilus paludosus R.Br. SO, EF

${ }^{\dagger} C$. robertsonii Benth. SO, EF

Calystegia marginata R.Br. SO, EF

$\$$ Carex berggrenii Petrie

C. capillacea Boott

C. chathamica C.B.Clarke ${ }_{\mathrm{IE}}$

C. edgariae Hamlin DP

$\doteqdot$ C. enysii Petrie $\mathrm{DP}$

C. lachenalii subsp. parkeri Petrie $\mathrm{DP}$

$\ddagger$ C. muelleri Petrie

C. tenuiculmis (Petrie) Heenan et de Lange

Celmisia hookeri Cockayne

$\dagger$ Centrolepis strigosa (R.Br.) Roem. et Schult. SO, EF

$\$$ Chionochloa vireta H.E.Connor $\mathrm{DP}$

$\$$ Clematis petriei Allan

Convolvulus fracto-saxosa Petrie

C. verecundus Allan

Coprosma intertexta G.Simpson

$\$ \dagger$ Corunastylis nuda (Hook.f.) D.L.Jones et M.A.Clem. SO, EF

$\$$ C. pumila (Hook.f.) D.L.Jones et M.A.Clem. SO, EF

${ }^{\dagger}$ Crassula helmsii (Kirk) Cockayne so

C. multicaulis (Petrie) A.P.Druce et Given ${ }_{\mathrm{EF}}$

C. ruamahanga A.P.Druce

$¥$ Deyeuxia youngii (Hook.f.) Buchanan

Doodia mollis Parris

Epilobium petraeum Heenan

E. purpuratum Hook.f.

$¥$ Euchiton ensifer (D.G.Drury) Holub DP

${ }^{\ddagger}$ E. paludosus (Petrie) Holub DP

${ }^{\dagger}$ Fimbristylis velata $\mathrm{R}$.Br. SO

Fuchsia procumbens R.Cunn. ex A.Cunn.

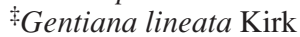

${ }^{\ddagger}$ Gingidia trifoliolata (Hook.f.) DP

Gnaphalium luteoalbum var. compactum Kirk DP, EF

Grammitis rawlingsii Parris

${ }^{\ddagger}$ G. rigida Hombr. DP, SO

$\ddagger$ Halocarpus kirkii (Parl.) Quinn ${ }_{\mathrm{RF}}$

Hebe annulata (Petrie) Cockayne et Allan

$H$. dilatata G.Simpson et J.S.Thomson

H. tairawhiti B.D.Clarkson et Garn.-Jones

${ }_{\sharp}$ Hierochloe fusca Zotov DP

$\stackrel{\$}{\sharp}$ ymenochilus tanypodus (D.L.Jones, Molloy et M.A. Clem.) D.L.Jones,

M.A.Clem. et Molloy EF

Apiaceae

Apiaceae

Orchidaceae

Poaceae

Poaceae

Poaceae

Ranunculaceae

Orchidaceae

Aspleniaceae

Aspleniaceae

Amaranthaceae

Blechnaceae

Ophioglossaceae

Orchidaceae

Orchidaceae

Convolvulaceae

Cyperaceae

Cyperaceae

Cyperaceae

Cyperaceae

Cyperaceae

Cyperaceae

Cyperaceae

Cyperaceae

Asteraceae

Centrolepidaceae

Poaceae

Ranunculaceae

Convolvulaceae

Convolvulaceae

Rubiaceae

Orchidaceae

Orchidaceae

Crassulaceae

Crassulaceae

Crassulaceae

Poaceae

Blechnaceae

Onagraceae

Onagraceae

Asteraceae

Asteraceae

Cyperaceae

Onagraceae

Gentianaceae

Apiaceae

Asteraceae

Grammitidaceae

Grammitidaceae

Podocarpaceae

Plantaginaceae

Plantaginaceae

Plantaginaceae

Poaceae

Orchidaceae 
$\ddagger$ H. tristis (Colenso) D.L.Jones, M.A.Clem. et Molloy HI, EF

$¥$ Hymenophyllum atrovirens Colenso

${ }^{\dagger}$ Hypolepis amaurorachis (Kunze) Hook. sO, EF

${ }^{\dagger} H$. dicksonioides (Kunze) Hook. SO, EF

$\ddagger$ Kirkianella novae-zelandiae (Hook.f.) Allan f. novae-zelandiae $\mathrm{HI}$

Korthalsella salicornioides (A.Cunn.) Tiegh. EF

¥Lagenifera lanata A.Cunn.

Lepidium naufragorum Garn.-Jones et D.A.Norton $\mathrm{ST}$

†eptinella dispersa (D.G.Lloyd) D.G.Lloyd et C.Webb subsp. dispersa

$\downarrow$ L. tenella (A.Cunn.) D.G.Lloyd et C.Webb

Libocedrus plumosa (D.Don) Sarg.

$\div$ Lindsaea viridis Colenso

$\$$ Macrothelypteris torresiana (Gaudich.) Ching SO, EF

Melicytus crassifolius (Hook.f.) F.Muell. sens. str. DP

Microlaena carsei Cheeseman

$¥$ Microsorum novae-zelandiae (Baker) Copel.

$\$$ Mimulus repens $\mathrm{R}$. Br. DP, so

Muehlenbeckia ephedroides Hook.f.

${ }^{\ddagger}$ Myosotis saxosa Hook.f. DP

$¥$ M. spathulata Forst.f. var. spathulata DP

$¥$ M. tenericaulis Petrie $\mathrm{DP}$

${ }^{\ddagger}$ Neopaxia erythrophylla Heenan DP

Olearia capillaris Buchanan

$O$. fragrantissima Petrie

$¥ O$. lineata (Kirk) Cockayne $\mathrm{RF}$

$\dagger$ Peperomia tetraphylla (G.Forst.) Hook. et Arn. so

$\dagger$ Picris angustifolia subsp. merxmuelleri Lack et S.Holzapfel ${ }_{\mathrm{EF}}$

¥Pimelea poppelwellii Petrie

$¥$ P. pseudolyallii Allan

† Pisonia brunoniana Endl. SO, HI

$¥$ Pittosporum ellipticum Kirk

$P$. pimeleoides R.Cunn. subsp. pimeleoides

$P$. virgatum Kirk

Plantago obconica Sykes

$\dagger$ Pleurosorus rutifolius (R.Br.) Fée so

$¥$ Poa incrassata Petrie

Pomaderris hamiltonii L.B.Moore $\mathrm{RC}$

Pseudopanax ferox Kirk $\mathrm{CD}, \mathrm{RF}$

$\stackrel{\square}{\square}$. macintyrei Cheeseman

Pterostylis cernua D.L.Jones, Molloy et M.A.Clem. EF

Ranunculus maculatus Cockayne et Allan

$¥ R$. simulans Garn.-Jones

${ }^{\ddagger}$ Raoulia beauverdii Cockayne

$¥$ Rytidosperma merum Connor et Edgar DP

$¥$ Scandia rosifolia (Hook.) J.W.Dawson DP

\$Schizaea dichotoma (L.) J.E.Sm. so

$¥$ Schizeilema pallidum (Kirk) Domin DP

$\$$ Senecio carnosulus (Kirk) C.Webb

S. dunedinensis Belcher $\mathrm{EF}$

S. marotiri C.J.Webb

S. repangae de Lange et B.G.Murray subsp. repangae $\mathrm{EF}$

†Solanum aviculare $\mathrm{f}$. latifolium G.T.S.Baylis

Stegostyla atradenia (D.L.Jones, Molloy et M.A.Clem.) D.L.Jones et M.A.Clem

\$Sticherus flabellatus (R.Br.) H.St.John so

$¥$ Streblus banksii (Cheeseman) C.Webb

$\dagger$ Tetragonia tetragonioides (Pallas) Kuntze $\mathrm{EF}$

\$Thelymitra formosa Colenso DP, EF

T. tholiformis Molloy et Hatch $\mathrm{EF}$

${ }^{\dagger}$ Thismia rodwayi F.Muell. DP, SO, EF

Tmesipteris sigmatifolia Chinnock

Townsonia deflexa Cheeseman
Orchidaceae

Hymenophyllaceae

Dennstaedtiaceae

Dennstaedtiaceae

Asteraceae

Santalaceae

Asteraceae

Brassicaceae

Asteraceae

Asteraceae

Cupressaceae

Dennstaedtiaceae

Thelypteridaceae

Violaceae

Poaceae

Polypodiaceae

Phrymaceae

Polygonaceae

Boraginaceae

Boraginaceae

Boraginaceae

Portulacaceae

Asteraceae

Asteraceae

Asteraceae

Piperaceae

Asteraceae

Thymelaeaceae

Thymelaeaceae

Nyctaginaceae

Pittosporaceae

Pittosporaceae

Pittosporaceae

Plantaginaceae

Aspleniaceae

Poaceae

Rhamnaceae

Araliaceae

Araliaceae

Orchidaceae

Ranunculaceae

Ranunculaceae

Asteraceae

Poaceae

Apiaceae

Schizaeaceae

Araliaceae

Asteraceae

Asteraceae

Asteraceae

Asteraceae

Solanaceae

Orchidaceae

Gleicheniaceae

Moraceae

Aizoaceae

Orchidaceae

Orchidaceae

Burmanniaceae

Psilotaceae

Orchidaceae 
$¥$ Traversia baccharoides Hook.f.

$¥$ Trichomanes colensoi Hook.f. DP

†Trisetum drucei Edgar $\mathrm{DP}$

\$ncinia elegans (Kük.) Hamlin

$\doteqdot$ U. longifructus (Kük.) Petrie

$U$. purpurata Petrie

$\ddagger$ U. sinclairii Boott

$\ddagger U$. viridis (C.B.Clarke) Edgar

Urtica aspera Petrie ${ }_{\mathrm{DP}}$

Wahlenbergia congesta (Cheeseman) N.E.Brown subsp. congesta
Asteraceae

Hymenophyllaceae

Poaceae

Cyperaceae

Cyperaceae

Cyperaceae

Cyperaceae

Cyperaceae

Urticaceae

Campanulaceae

\section{Range Restricted (373)}

Taxa whose distribution is naturally confined to specific substrates (e.g., ultramafic rock), habitats (e.g., high alpine fell field), or geographic areas (e.g., subantarctic islands). Range Restricted taxa occupy an area of $<100 \mathrm{~km}^{2}$ and within that area they are under no obvious or immediate threat. However, because of their distribution they are naturally susceptible to extirpation.

¥Abrotanella muscosa Kirk IE

Asteraceae

$¥$ A. patearoa Heads

$¥$ A. rostrata Swenson

Asteraceae

$¥$ A. rosulata Hook.f. OL, IE

$¥$ A. spathulata Hook.f.

Asteraceae

Acaena microphylla var. pauciglochidiata Bitter

Asteraceae

Asteraceae

A. minor Allan

$\ddagger$ A. tesca B.H.Macmill.

Achnatherum petriei (Buchanan) S.W.L.Jacobs et J.Everett CD

$¥$ Aciphylla cartilaginea Petrie

$¥$ A. congesta Cheeseman

$¥$ A. crosby-smithii Petrie

$¥$ A. dissecta (Kirk) W.R.B.Oliv.

$¥$ A. lecomtei J.W.Dawson

A. leighii Allan

A. montana var. gracilis (W.R.B.Oliv.) J.W.Dawson

$¥$ A. spedenii Cheeseman

A. squarrosa var. flaccida Kirk

A. stannensis J.W.Dawson

$¥$ A. takahea W.R.B.Oliv.

$¥$ A. traillii Kirk

A. trifoliolata Petrie DP

$¥$ Agrostis subulata Hook.f.

Anisotome acutifolia (Kirk) Cockayne $\mathrm{OL}, \mathrm{IE}$

$¥$ A. antipoda Hook.f. OL

$¥$ A. latifolia Hook.f.

$¥$ A. lyallii Hook.f.

$\doteqdot$ Apium prostratum subsp. denticulatum P.S.Short $\mathrm{IE}$

$\because$ Arachniodes aristata (G.Forst.) Tindale so, OL

Argyrotegium nitidulum (Hook.f.) J.M.Ward et Breitw. so

${ }^{\ddagger}$ Asplenium chathamense Brownsey IE

${ }^{*}$ Asplenium shuttleworthianum Kunze so, OL

$¥$ Astelia nivcola var. moriceae L.B.Moore

$\dagger$ Atriplex billardierei (Moq.) Hook.f. CD, TO, HI

Baumea complanata (Bergg.) Blake $\mathrm{HI}$

Rosaceae

Rosaceae

Rosaceae

Poaceae

Apiaceae

Apiaceae

Apiaceae

Apiaceae

Apiaceae

Apiaceae

Apiaceae

Apiaceae

Apiaceae

Apiaceae

Apiaceae

Apiaceae

Apiaceae

Poaceae

Apiaceae

Apiaceae

Apiaceae

Apiaceae

Apiaceae

Dryopteridaceae

Asteraceae

Aspleniaceae

Aspleniaceae

Asteliaceae

Amaranthaceae

Cyperaceae

Brachyglottis arborescens W.R.B.Oliv. IE

B. bifistulosa (Hook.f.) B.Nord. DP

B. compacta (Kirk) B.Nord. OL

$¥$ B. myrianthos (Cheeseman) D.G.Drury

B. pentacopa (D.G.Drury) B.Nord. OL

Asteraceae

Asteraceae

Asteraceae

Asteraceae

$\doteqdot$ B. stewartiae (J.B.Armstr.) B.Nord. DP

Asteraceae

Asteraceae

B. turneri (Cheeseman) C.Webb

Asteraceae

Brachyscome humilis G.Simpson et J.S.Thomson DP

B. linearis (Petrie) Druce $\mathrm{DP}$

$₫$ Bulbinella rossii (Hook.f.) Cheeseman

Asteraceae

Asteraceae

Asparagaceae 
$\$$ B. gibbsii Cockayne var. gibbsii

B. talbotii L.B.Moore DP, OL

$\ddagger$ Callitriche aucklandica R.Mason $\mathrm{IE}$

${ }^{\ddagger}$ C. petriei subsp. chathamensis R.Mason $\mathrm{IE}$

+ Canavalia rosea (Sw.) DC. SO, OL

Cardamine lacustris (Garn.-Jones et P.N.Johnson) Heenan EF

Carex albula Allan

$\ddagger$ C. decurtata Cheeseman

$\ddagger$ C. devia Cheeseman

C. elingamita Hamlin $\mathrm{RC}, \mathrm{IE}$

$\ddagger$ C. filamentosa Petrie

$\ddagger$ C. fretalis Hamlin DP

$\ddagger$ C. impexa K.A.Ford

$¥$ C. kermadecensis Petrie IE

C. ophiolithica de Lange et Heenan $\mathrm{CD}, \mathrm{ST}, \mathrm{OL}$

$\ddagger$ C. pleiostachys C.B.Clarke

$\ddagger$ C. pterocarpa Petrie

$\ddagger$ C. rubicunda Petrie

$\ddagger$ C. tahoata Hamlin

$\ddagger$ C. trachycarpa Cheeseman

C. traversii Kirk

C. uncifolia Cheeseman

C. ventosa C.B.Clarke $\mathrm{IE}$

Carmichaelia appressa G.Simpson $\mathrm{OL}$

C. astonii G.Simpson

C. compacta Petrie

C. torulosa (Kirk) Heenan $\mathrm{ST}, \mathrm{RF}$

Cassinia amoena Cheeseman $\mathrm{CD}$, ST, OL

Celmisia adamsii var. adamsii Cheeseman

C. adamsii var. rugulosa Cheeseman $\mathrm{OL}$

$\ddagger$ C. clavata G.Simpson et G.Thomson ${ }_{\mathrm{IE}}$

¥. cockayniana Petrie

C. cordatifolia Buchanan var. cordatifolia

$¥$ C. gibbsii Cheeseman

$¥$ C. glandulosa var. latifolia Cockayne $\mathrm{OL}$

C. inaccessa Given DP

C. insignis Martin $\mathrm{ST}$

$\$$ C. lindsayi Hook.f.

C. mackaui Raoul

C. macmahonii var. hadfieldii Martin

$\ddagger$ C. major var. brevis Allan $\mathrm{OL}$

$\ddagger C$. markii Given et W.R.Lee

C. morganii Cheeseman

C. philocremna Given

$\$$ C. polyvena G.Simpson et G.Thomson

$\ddagger$ C. rigida (Kirk) Cockayne

C. rutlandii Kirk

C. spectabilis subsp. lanceolata (Hook.f.) D.R.Given

C. spedenii G.Simpson

C. thomsonii Cheeseman $\mathrm{OL}$

$\dagger$ Cenchrus caliculatus Cav. TO, OL

Centrolepis minima Kirk

¥Chionochloa acicularis Zotov

$\ddagger$ C. antarctica (Hook.f.) Zotov

$¥$ C. beddiei Zotov

$\$$ C. bromoides (Hook.f.) Zotov

$\ddagger$ C. crassiuscula (Kirk) Zotov subsp. crassiuscula

$\ddagger$ C. crassiuscula subsp. directa Connor

$\ddagger$ C. defracta Connor

C. flavicans f. temata Connor $\mathrm{OL}$

$\ddagger$ C. juncea Zotov
Asparagaceae

Asparagaceae

Plantaginaceae

Plantaginaceae

Fabaceae

Brassicaceae

Cyperaceae

Cyperaceae

Cyperaceae

Cyperaceae

Cyperaceae

Cyperaceae

Cyperaceae

Cyperaceae

Cyperaceae

Cyperaceae

Cyperaceae

Cyperaceae

Cyperaceae

Cyperaceae

Cyperaceae

Cyperaceae

Cyperaceae

Fabaceae

Fabaceae

Fabaceae

Fabaceae

Asteraceae

Asteraceae

Asteraceae

Asteraceae

Asteraceae

Asteraceae

Asteraceae

Asteraceae

Asteraceae

Asteraceae

Asteraceae

Asteraceae

Asteraceae

Asteraceae

Asteraceae

Asteraceae

Asteraceae

Asteraceae

Asteraceae

Asteraceae

Asteraceae

Asteraceae

Asteraceae

Poaceae

Centrolepidaceae

Poaceae

Poaceae

Poaceae

Poaceae

Poaceae

Poaceae

Poaceae

Poaceae

Poaceae 
$\ddagger$ C. lanea Connor

$¥$ C. ovata (Buchanan) Zotov

$\$$ C. rubra subsp. rubra var. inermis Connor $\mathrm{OL}$

${ }^{\ddagger}$ C. spiralis Zotov

Chionohebe glabra (Cheeseman) Heads DP

C. myosotoides (Ashwin) B.Briggs et Ehrend. DP

$¥$ Colobanthus hookeri Cheeseman

$\$$ C. squarrosus subsp. drucei Sneddon

$\pitchfork$ C. squarrosus Cheeseman subsp. squarrosus

Coprosma distantia (de Lange et R.O.Gardner) de Lange $\mathrm{CD}$, OL

C. neglecta Cheeseman

C. talbrockiei L.B.Moore et R.Mason

Cordyline kaspar W.R.B.Oliv.

Corokia macrocarpa Kirk CD, IE

Coriaria pottsiana W.R.B.Oliv.

$\$$ Craspedia robusta var. pedicellata (Kirk) Allan

Cyathea kermadecensis W.R.B.Oliv. RC, IE

$\ddagger$ C. milnei Hook.f. IE

†amnamenia vernicosa (Hook.f.) D.R.Given

Deyeuxia lacustris Edgar ${ }_{\mathrm{DP}}$

Dichelachne lautumia Edgar et Connor ${ }_{\mathrm{ST}}$

$\dagger$ Dicranopteris linearis (Burm.f.) Underw. so

$¥$ Disphyma australe subsp. stricticaule Chinnock OL, IE

$\ddagger$ D. papillatum Chinnock IE

${ }^{\ddagger}$ Doodia milnei (Carr.) Parris OL, IE

$\ddagger$ Dracophyllum arboreum Cockayne $\mathrm{IE}$

$¥ D$. densum W.R.B.Oliv.

D. marmoricola S.Venter

D. ophioliticum S.Venter

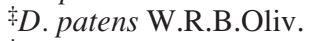

D. trimorphum W.R.B.Oliv.

$¥ D$. uniflorum var. frondosum G.Simpson $\mathrm{PD}$

Elingamita johnsonii G.T.S.Baylis OL, IE

¥Elymus apricus Á.Löve et Connor

$\ddagger$ E. falcis Connor

$\ddagger$ E. sacandros Connor

${ }^{\ddagger}$ Epilobium astonii (Allan) Raven et Raven

$\ddagger$ E. brevipes Hook.f.

$\ddagger$ E. confertifolium Hook.f.

$\ddagger$ E. forbesii Allan

E. margaretiae Brockie

$\ddagger$ E. wilsonii Petrie

\#uphrasia drucei M.B.Ashwin

$\ddagger$ E. repens Hook.f.

$\ddagger$ E. wettsteiniana du Rietz

Ewartiothamnus sinclairii (Hook.f.) Anderb.

Festuca coxii (Petrie) Hack. IE

F. luciarum Connor DP

$\stackrel{+}{*}$. matthewsii subsp. pisamontis Connor

$¥$ F. ultramafica Connor OL

Geniostoma ligustrifolium var. crassum Cheeseman CD, ST, OL

${ }^{\ddagger}$ G. ligustrifolium var. maius Cheeseman $\mathrm{RC}$, IE

Gentiana antarctica Kirk OL, IE

G. antipoda Kirk OL, IE

$¥$ G. astonii Petrie $\mathrm{ST}$

G. bellidifolia var. magnifica Kirk ST

$\$ G$. chathamica Cheeseman $\mathrm{IE}$

$\$$ G. cerina Hook.f. IE

$\ddagger$ G. concinna Hook.f. IE

$\ddagger$ G. filipes Cheeseman

G. gibbsii Petrie $\mathrm{OL}$
Poaceae

Poaceae

Poaceae

Poaceae

Plantaginaceae

Plantaginaceae

Caryophyllaceae

Caryophyllaceae

Caryophyllaceae

Rubiaceae

Rubiaceae

Rubiaceae

Asparagaceae

Argophyllaceae

Coriariaceae

Asteraceae

Cyatheaceae

Cyatheaceae

Asteraceae

Poaceae

Poaceae

Gleicheniaceae

Aizoaceae

Aizoaceae

Blechnaceae

Ericaceae

Ericaceae

Ericaceae

Ericaceae

Ericaceae

Ericaceae

Ericaceae

Myrsinaceae

Poaceae

Poaceae

Poaceae

Onagraceae

Onagraceae

Onagraceae

Onagraceae

Onagraceae

Onagraceae

Orobanchaceae

Orobanchaceae

Orobanchaceae

Asteraceae

Poaceae

Poaceae

Poaceae

Poaceae

Loganiaceae

Loganiaceae

Gentianaceae

Gentianaceae

Gentianaceae

Gentianaceae

Gentianaceae

Gentianaceae

Gentianaceae

Gentianaceae

Gentianaceae 
G. lilliputiana C.Webb $\mathrm{EF}$

Geranium traversii Hook.f. IE

Geum divergens Cheeseman

$¥$ Gingidia enysii (Kirk) J.W.Dawson DP

$\ddagger$ G. flabellata (Kirk) J.W.Dawson $\mathrm{IE}$

$\ddagger$ Haastia pulvinaris var. minor Laing

${ }^{*}$. recurva var. wallii Cockayne $\mathrm{DP}$

Haloragis erecta subsp. cartilaginea (Cheeseman) Orchard $\mathrm{CD}$, ST, OL

Hebe acutiflora Cockayne $\mathrm{PD}$

H. adamsii (Cheeseman) Cockayne et Allan OL

$¥$ H. amplexicaulis (J.B.Armstr.) Cockayne et Allan f. amplexicaulis

H. amplexicaulis $\mathrm{f}$. hirta Garn.-Jones et Molloy

H. arganthera Garn.-Jones, Bayly, W.G.Lee et Rance

$¥ H$. benthamii (Hook.f.) Cockayne et Allan

$¥$ H. biggarii Cockayne

$H$. brevifolia (Cheeseman) de Lange $\mathrm{CD}, \mathrm{ST}$, OL

H. calcicola Bayly et Garn.-Jones

$¥$ H. carnosula (Hook.f.) Cockayne

$¥$ H. chathamica (Buchanan) Cockayne et Allan $\mathrm{IE}$

H. dieffenbachii (Benth.) Cockayne et Allan $\mathrm{IE}$

H. elliptica var. crassifolia Cockayne et Allan

${ }^{*}$. evenosa (Petrie) Cockayne et Allan

H. gibbsii (Kirk) Cockayne et Allan DP

$\$$ H. imbricata Cockayne et Allan

H. insularis (Cheeseman) Cockayne et Allan IE

$¥$ H. macrocalyx (J.B.Armstr.) G.Simpson var. macrocalyx

$\ddagger$ H. obtusata (Cheeseman) Cockayne et Allan

H. ochracea M.B.Ashwin

H. pareora Garn.-Jones et Molloy

$\$$ H. pauciflora G.Simpson et J.S.Thomson ${ }_{\mathrm{DP}}$

${ }^{\ddagger} H$. pubescens subsp. rehuarum Bayly et de Lange $\mathrm{IE}$

$H$. pubescens subsp. sejuncta Bayly et de Lange

H. ramosissima G.Simpson et J.S.Thomson

${ }^{*}$. rigidula (Cheeseman) Cockayne et Allan var. rigidula

$¥ H$. rigidula var. sulcata Bayly et Kellow DP

$¥$ H. stenophylla var. hesperia Bayly et Garn.-Jones

${ }^{\ddagger} H$. stenophylla var. oliveri Bayly et Garn.-Jones OL, IE

H. townsonii (Cheeseman) Cockayne et Allan

$¥ H$. tumida (Kirk) Cockayne et Allan

${ }^{\ddagger}$ H. urvilleana W.R.B.Oliv.

Helichrysum plumeum Allan

$H$. selago var. tumidum Cheeseman

$¥$ Hierochloe brunonis Hook.f.

$\$$ Hoheria equitum Heads

$¥$ Homalanthus polyandrus (Hook.f.) Cheeseman OL

$\doteqdot$ Imperata cheesemanii Hack. OL, IE

†Ipomoea pes-caprae subsp. brasiliensis (L.) Ooststr. so

$\dagger$ Isolepis crassiuscula Hook.f. so

$\dagger$ Juncus scheuchzerioides Gaudich. so

Kirkianella novae-zelandiae f. glauca Allan $\mathrm{ST}, \mathrm{HI}$

$¥$ Kunzea ericoides var. microflora (G.Simpson) W.Harris

K. sinclairii (Kirk) W.Harris $\mathrm{IE}$

Lachnagrostis ammobia Edgar DP

$¥$ L. leptostachys (Hook.f.) Zotov

${ }^{\ddagger}$ L. pilosa subsp. nubifera Edgar

$¥$ L. tenuis (Cheeseman) Edgar

Leptecophylla robusta (Hook.f.) Weiller ${ }_{\mathrm{IE}}$

Leptinella albida (D.G.Lloyd) D.G.Lloyd et C.Webb

$¥$ L. atrata subsp. luteola (D.G.Lloyd) D.G.Lloyd et C.Webb

L. calcarea (D.G.Lloyd) D.G.Lloyd et C.Webb

L. dispersa subsp. rupestris (D.G.Lloyd) D.G.Lloyd et C.Webb ${ }_{\mathrm{RF}}$
Gentianaceae

Geraniaceae

Rosaceae

Apiaceae

Apiaceae

Asteraceae

Asteraceae

Haloragaceae

Plantaginaceae

Plantaginaceae

Plantaginaceae

Plantaginaceae

Plantaginaceae

Plantaginaceae

Plantaginaceae

Plantaginaceae

Plantaginaceae

Plantaginaceae

Plantaginaceae

Plantaginaceae

Plantaginaceae

Plantaginaceae

Plantaginaceae

Plantaginaceae

Plantaginaceae

Plantaginaceae

Plantaginaceae

Plantaginaceae

Plantaginaceae

Plantaginaceae

Plantaginaceae

Plantaginaceae

Plantaginaceae

Plantaginaceae

Plantaginaceae

Plantaginaceae

Plantaginaceae

Plantaginaceae

Plantaginaceae

Plantaginaceae

Asteraceae

Asteraceae

Poaceae

Malvaceae

Euphorbiaceae

Poaceae

Convolvulaceae

Cyperaceae

Juncaceae

Asteraceae

Myrtaceae

Myrtaceae

Poaceae

Poaceae

Poaceae

Poaceae

Ericaceae

Asteraceae

Asteraceae

Asteraceae

Asteraceae 
$\ddagger$ L. lanata Hook.f.

Asteraceae

$\ddagger$ L. minor Hook.f.

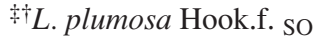

$¥$ L. potentillina F.Muell.

L. pyrethrifolia var. linearifolia (Cheeseman) D.G.Lloyd et C.Webb OL

$\ddagger$ L. traillii subsp. puchella (Kirk) D.G.Lloyd et C.Webb

${ }^{\ddagger}$ L. traillii (Kirk) D.G.Lloyd et C.Webb subsp. traillii

$¥$ Leucogenes neglecta Molloy

${ }^{\dagger}$ Leucopogon parviflorus (Andrews) Lindl. OL, so

L. xerampelinus de Lange, Heenan et M.I.Dawson $\mathrm{CD}, \mathrm{ST}$, OL

$¥$ Lignocarpa diversifolia (Cheeseman) J.W.Dawson

Luzula crenulata Buchanan

${ }^{\ddagger}$ L. traversii var. tenuis Edgar $\mathrm{DP}$

Macropiper excelsum subsp. peltatum f. delangei R.O.Gardner ${ }_{\mathrm{RC}} \mathrm{IE}$

$¥$ M. excelsum subsp. peltatum R.O.Gardner f. peltatum

${ }^{+}$M. excelsum subsp. psittacorum (Endl.) Sykes SO, OL

M. melchior Sykes IE

$¥$ Melicytus chathamicus (F.Muell.) Garn.-Jones IE

M. drucei Molloy et B.D.Clarkson $\mathrm{CD}, \mathrm{RC}$, OL

$\ddagger$ M. obovatus (Kirk) Garn.-Jones sens. str.

Meryta sinclairii (Hook.f.) Seem

$¥$ Metrosideros kermadecensis W.R.B.Oliv. IE

$¥$ Mitrasacme montana var. helmsii Kirk

$¥$ Myoporum kermadecense Sykes IE

$\ddagger$ Myosotis antarctica Hook.f. IE

$\$$ M. arnoldii L.B.Moore

M. capitata Hook.f. DP

$\ddagger$ M. concinna Cheeseman

$\ddagger$. eximia Petrie

$\ddagger$ M. explanata Cheeseman $\mathrm{DP}$

M. laeta Cheeseman OL

$\ddagger$ M. matthewsii L.B.Moore DP, EF

$\ddagger$ M. monroi Cheeseman $\mathrm{OL}$

M. oreophila Petrie $\mathrm{HI}$, EF

$¥ M$. rakiura L.B.Moore

Myrsine coxii Cockayne ${ }_{\mathrm{RF}}$, IE

$\ddagger$ M. kermadecensis Cheeseman OL, IE

M. oliveri Allan OL, IE

$¥$ Neopaxia racemosa (Buchanan) Heenan $\mathrm{OL}$

${ }^{*}$ Nephrolepis hirsutula (G.Forst.) C.Presl SO, OL

Olearia allomii Kirk OL, IE

O. chathamica Kirk IE

$\ddagger$ O. crosby-smithiana Petrie

$¥ O$. semidentata Decne. IE

O. traversii (F.Muell.) Hook.f. CD, RC, IE

$\$$ Oreoporanthera alpina (Cheeseman) Orchard

¥Ourisia confertifolia Arroyo

O. goulandiana Arroyo DP

$¥ O$. remotifolia Arroyo

O. spathulata Arroyo DP

$¥$ Pachycladon crenatus Phillipson

$\ddagger P$. wallii (Carse) Heenan et A.Mitch.

Pachystegia rufa Molloy $\mathrm{CD}$, ST

Parsonsia praeruptis Heads et de Lange $\mathrm{CD}$, $\mathrm{OL}$

${ }^{\dagger}$ Petalochilus alatus (R.Br.) D.L.Jones et M.A.Clem. DP, TO

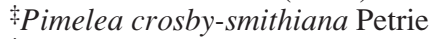

$\$ P$. microphylla Colenso

$\ddagger$ P. suteri Kirk

$¥$ Pittosporum fairchildii Cheeseman $\mathrm{IE}$

P. pimeleoides subsp. maius (Cheeseman) R.C.Cooper $\mathrm{CD}$, ST, OL

¥Plantago aucklandica Hook.f. IE

Asteraceae

Asteraceae

Asteraceae

Asteraceae

Asteraceae

Asteraceae

Asteraceae

Ericaceae

Ericaceae

Apiaceae

Juncaceae

Juncaceae

Piperaceae

Piperaceae

Piperaceae

Piperaceae

Violaceae

Violaceae

Violaceae

Araliaceae

Myrtaceae

Loganiaceae

Myoporaceae

Boraginaceae

Boraginaceae

Boraginaceae

Boraginaceae

Boraginaceae

Boraginaceae

Boraginaceae

Boraginaceae

Boraginaceae

Boraginaceae

Boraginaceae

Myrsinaceae

Myrsinaceae

Myrsinaceae

Portulacaceae

Nephrolepidaceae

Asteraceae

Asteraceae

Asteraceae

Asteraceae

Asteraceae

Euphorbiaceae

Plantaginaceae

Plantaginaceae

Plantaginaceae

Plantaginaceae

Brassicaceae

Brassicaceae

Asteraceae

Apocynaceae

Orchidaceae

Thymelaeaceae

Thymelaeaceae

Thymelaeaceae

Pittosporaceae

Pittosporaceae

Plantaginaceae 
P. spathulata subsp. picta (Colenso) Sykes

$¥ P$. triantha Spreng. IE

$\$$ Pleurophyllum criniferum Hook.f.

${ }^{\dagger} P$. hookeri Buchanan

$\ddagger P$. speciosum Hook.f.

$¥$ Poa acicularifolia Buchanan subsp. acicularifolia

$\$$ P. acicularifolia subsp. ophitalis Edgar

$¥ P$. anceps subsp. polyphylla (Hack.) Edgar

$¥ P$. antipoda Petrie

$¥ P$. aucklandica Petrie subsp. aucklandica $\mathrm{IE}$

$¥ P$. aucklandica subsp. campbellensis (Petrie) Edgar $\mathrm{IE}$

P. aucklandica subsp. rakiura Edgar $\mathrm{DP}, \mathrm{OL}, \mathrm{IE}$

$¥ P$. chathamica Petrie $\mathrm{IE}$

${ }^{\dagger} P$. foliosa (Hook.f.) Hook.f.

$P$. pygmaea Buchanan

$¥ P$. ramosissima Hook.f.

P. senex Edgar

$\ddagger$ P. tennantiana Petrie

P. xenica Edgar et Connor

Pomaderris paniculosa subsp. novae-zelandiae (L.B.Moore) N.G.Walsh

$¥ P$. rugosa Cheeseman

${ }_{\dagger}$ Poranthera microphylla Brong. so

$¥$ Pseudopanax chathamicus Kirk $\mathrm{IE}$

$\ddagger P$. gilliesii Kirk

$\ddagger$ P. kermadecensis (W.R.B.Oliv.) Phillipson RC, OL, IE

$¥$ Pterostylis silvicultrix (F.Muell.) Molloy, D.L.Jones et M.A.Clem. IE

$¥$ Puccinellia walkeri subsp. antipoda (Petrie) Edgar $\mathrm{OL}, \mathrm{IE}$

$\ddagger P$. walkeri (Kirk) Allan subsp. walkeri

Rachelia glaria J.M.Ward et Breitw. DP

$¥$ Ranunculus brevis Garn.-Jones

$R$. godleyanus Hook.f. CD

R. grahamii Petrie

$R$. haastii subsp. piliferus F.J.F.Fisher

$\stackrel{\sim}{*}$. kirkii Petrie

$\ddagger R$. mirus Garn.-Jones

$\stackrel{+}{*}$. pinguis Hook.f.

$R$. recens var. lacustris G.Simpson

$R$. scrithalis Garn.-Jones

$R$. stylosus H.D.Wilson et Garn.-Jones

$\sharp R$. subscaposus Hook.f. OL, IE

R. viridis H.D.Wilson et Garn.-Jones DP, OL

Raoulia cinerea Petrie

${ }^{\ddagger}$. goyenii Kirk

$¥ R$. hectorii var. mollis Buchanan

$\$ R$. petriensis Kirk

$¥ R$. rubra Buchanan

$\ddagger$ Rhopalostylis cheesemanii Becc. IE

${ }^{\dagger}$ Rumex neglectus Kirk ${ }_{\mathrm{HI}}$

${ }^{\dagger}$ Rytidosperma nudum (Hook.f.) Connor et Edgar DP

$R$. petrosum Connor et Edgar

+Scaevola gracilis Hook.f. OL, TO

¥Schizeilema reniforme (Hook.f.) Domin

+ Schoenus fluitans Hook.f. so

$¥$ Senecio glaucophyllus subsp. basinudus Ornduff

${ }^{\ddagger}$ S. glaucophyllus Cheeseman subsp. glaucophyllus

S. hauwai Sykes ST

S. lautus var. esperensis Sykes OL, EF

${ }^{\ddagger}$ S. radiolatus subsp. antipodus (Kirk) C.Webb OL, EF, IE

$¥$ S. radiolatus F.Muell. subsp. radiolatus $\mathrm{EF}$, IE

S. repangae subsp. pokohinuensis de Lange et B.G.Murray OL, EF, IE

${ }^{\sharp}$ S. sterquilinus Ornduff ${ }_{\mathrm{HI}}, \mathrm{EF}$
Plantaginaceae

Plantaginaceae

Asteraceae

Asteraceae

Asteraceae

Poaceae

Poaceae

Poaceae

Poaceae

Poaceae

Poaceae

Poaceae

Poaceae

Poaceae

Poaceae

Poaceae

Poaceae

Poaceae

Poaceae

Rhamnaceae

Rhamnaceae

Euphorbiaceae

Araliaceae

Araliaceae

Araliaceae

Orchidaceae

Poaceae

Poaceae

Asteraceae

Ranunculaceae

Ranunculaceae

Ranunculaceae

Ranunculaceae

Ranunculaceae

Ranunculaceae

Ranunculaceae

Ranunculaceae

Ranunculaceae

Ranunculaceae

Ranunculaceae

Ranunculaceae

Asteraceae

Asteraceae

Asteraceae

Asteraceae

Asteraceae

Arecaceae

Polygonaceae

Poaceae

Poaceae

Goodeniaceae

Araliaceae

Cyperaceae

Asteraceae

Asteraceae

Asteraceae

Asteraceae

Asteraceae

Asteraceae

Asteraceae

Asteraceae 
Simplicia buchananii (Zotov) Zotov DP

¥Sophora longicarinata G.Simpson et J.S.Thomson

†. molloyi Heenan et de Lange

Sporadanthus ferrugineus de Lange, Heenan et B.D.Clarkson CD, ST, HI

S. traversii (F.Muell.) F.Muell. CD, ST, IE

†prengelia incarnata Sm.

†tellaria decipiens Hook.f. var. decipiens

S. decipiens var. angustata Kirk IE

Stenostachys deceptorix Connor DP

†Stilbocarpa lyallii J.B.Armstr. RC

$\$ S$. polaris (Hombr. et Jacq.) Gray

$¥$ S. robusta (Kirk) Cockayne OL, IE

¥Streblus smithii (Cheeseman) Corner IE

$\$$ Trisetum serpentinum Edgar et A.P.Druce

Wahlenbergia akaroa J.A.Petterson

$\pitchfork W$. albomarginata subsp. flexilis (Petrie) J.A.Petterson

$\ddagger W$. albomarginata subsp. olivina J.A.Petterson

$\ddagger W$. cartilaginea Hook.f.

$\ddagger W$. matthewsii Cockayne DP

W. pygmaea subsp. drucei J.A.Petterson

Xeronema callistemon f. bracteosa (L.B.Moore) de Lange et E.K.Cameron OL, IE

${ }^{\dagger}$. callistemon W.R.B.Oliv. f. callistemon $\mathrm{IE}$
Poaceae

Fabaceae

Fabaceae

Restionaceae

Restionaceae

Ericaceae

Caryophyllaceae

Caryophyllaceae

Poaceae

Araliaceae

Araliaceae

Araliaceae

Moraceae

Poaceae

Campanulaceae

Campanulaceae

Campanulaceae

Campanulaceae

Campanulaceae

Campanulaceae

Xeronemataceae

Xeronemataceae

\section{Non-resident Native (26)}

Taxa whose natural presence in New Zealand is either sporadic or temporary (1. Vagrant) or they have succeeded in recently ( $\leq 50$ years) establishing themselves beyond their point of introduction (2. Coloniser).

\section{Vagrant (16)}

Taxa whose occurrences, though natural, are sporadic and typically transitory. Most (if not all) fail to establish themselves beyond their point of arrival because of reproductive failure or for specific ecological reasons. Listed here also are those vagrants which are currently known in the New Zealand Botanical Region only from historic herbarium specimens but which remain extant in their country of origin, and so retain the potential to re-establish themselves.

†Adiantum formosum R.Br. so

$\dagger$ Atriplex australasica Moq. so

$+{ }^{+}$Chiloglottis trapeziformis Fitz. EW, SO

${ }^{\dagger}$ Simpliglottis valida (D.L.Jones) D.L.Szlachekto so

$\dagger$ Doodia aspera A.Rich. EW, SO

${ }^{\dagger}$ Gratiola pubescens Benth. so

${ }^{\dagger}$ Lepturus repens var. cinereus (Burcham) Fosberg so

$\dagger$ Mazus pumilio $\mathrm{R}$.Br. so

$\uparrow$ Muellerina celastroides (Schult.f. et J.H.Schult.bis) Tiegh. so

${ }^{\dagger}$ Paracaleana minor (R.Br.) Blaxell so

${ }^{\dagger}$ Peperomia blanda (Jacq.) Humb., Bonpl. et Kunth so

$\dagger$ Picris angustifolia DC. subsp. angustifolia $\mathrm{SO}$

${ }_{\dagger}^{\dagger}$ Pterostylis nutans $\mathrm{R}$.Br. so

\#Senecio australis (Forst.f.) Willd. so

$\dagger$ Sticherus tener (R.Br.) Ching so

${ }^{\dagger}$ Wilsonia backhousei Hook.f. so
Pteridaceae
Amaranthaceae
Orchidaceae
Orchidaceae
Blechnaceae
Plantaginaceae
Poaceae
Phrymaceae
Loranthaceae
Orchidaceae
Piperaceae
Asteraceae
Orchidaceae
Asteraceae
Gleicheniaceae
Convolvulaceae

\section{Coloniser (10)}

Taxa which have arrived without direct or indirect human assistance and which have been successfully reproducing in the wild for $<50$ years.

${ }^{*}$ Achyranthes aspera L. sens. lat. DP, SO

$\dagger$ Atriplex cinerea Poir. so

$\dagger$ Cryptostylis subulata (Labill.) Rchb.f. so

$\dagger$ Diplodium alveatum (Garnet) D.L.Jones et M.A.Clem. so

$\dagger$ Epilobium gunnianum Hausskn. so

$\dagger$ Gratiola pedunculata $\mathrm{R}$.Br. so

$\dagger$ Plectranthus parviflorus Willd. so

†Sirpus polystachyus F.Muell. so

$\dagger$ Thelymitra malvina M.A.Clem., D.L.Jones et Molloy so

${ }^{\dagger}$ T. matthewsii Cheeseman то
Amaranthaceae

Amaranthaceae

Orchidaceae

Orchidaceae

Onagraceae

Plantaginaceae

Lamiaceae

Cyperaceae

Orchidaceae

Orchidaceae 
Data Deficient (45)

Taxa that are suspected but not definitely known to belong to any of the above categories due to a lack of current information about their present-day distribution and abundance. It is hoped that listing such taxa will stimulate research to find out the true category or threat. For a fuller definition see Molloy et al. (2002).

$\ddagger$ Cardamine bilobata Kirk

Carex allanii Hamlin DP

C. carsei Petrie

${ }^{\ddagger}$ C. druceana Hamlin

$\$$ C. raoulii Boott

Centipeda aotearoana N.G.Walsh

$\dagger$ Chenopodium detestans Kirk TO

C. pusillum Hook.f.

$\$$ Colobanthus brevisepalus Kirk

Crassula mataikona A.P.Druce

Deschampsia pusilla Petrie

¥Elymus tenuis (Buchanan) Á.Löve et Connor

$\dagger$ Epilobium hirtigerum A.Cunn. DP, SO, HI

E. pictum Petrie

$\ddagger$ E. vernicosum Cheeseman

Euchiton polylepis (D.G.Drury) Breitw. et J.M.Ward

\#uphrasia integrifolia Petrie

${ }^{\ddagger}$ Geranium microphyllum Hook.f. sens. str.

$\ddagger$ Geum albiflorum (Hook.f.) Cheeseman $\mathrm{IE}$

$\dagger^{\dagger}$ Grammitis gunnii Parris DP, SO

Gunnera densiflora Hook.f.

Hebe matthewsii (Cheeseman) Cockayne

Koeleria riguorum Edgar et Gibb

Lachnagrostis uda Edgar

+ Lagenifera montana Hook.f. DP

$\$$ Lepidium desvauxii Thell. so

$\dagger$ Lepilaena bilocularis Kirk so

$¥$ Libertia cranwelliae Blanchon, B.G.Murray et Braggins

Myosotis glabrescens L.B.Moore

M. laingii Cheeseman

M. petiolata var. pottsiana L.B.Moore

$¥$ M. suavis Petrie

$\ddagger$ M. uniflora Hook.f.

Nematoceras rivularis Hook.f. sens. str.

$¥$ Neopaxia lineariifolia Heenan

$¥$ Olearia angulata Kirk

$\ddagger$ Pachystegia minor (Cheeseman) Molloy

${ }_{\dagger}^{\dagger}$ Polygonum plebeium R.Br. so

$¥$ Pterostylis auriculata Colenso

$P$. irwinii D.L.Jones, Molloy et M.A.Clem.

P. porrecta D.L.Jones, Molloy et M.A.Clem.

$¥$ Schizeilema allanii Cheeseman

$¥$ Stenostachys laevis (Petrie) Connor

¥Vittadinia australis Hook.f.

Zotovia acicularis Edgar et Connor
Brassicaceae

Cyperaceae

Cyperaceae

Cyperaceae

Cyperaceae

Asteraceae

Amaranthaceae

Amaranthaceae

Caryophyllaceae

Crassulaceae

Poaceae

Poaceae

Onagraceae

Onagraceae

Onagraceae

Asteraceae

Orobanchaceae

Geraniaceae

Rosaceae

Grammitidaceae

Gunneraceae

Plantaginaceae

Poaceae

Poaceae

Asteraceae

Brassicaceae

Potamogetonaceae

Iridaceae

Boraginaceae

Boraginaceae

Boraginaceae

Boraginaceae

Boraginaceae

Orchidaceae

Portulacaceae

Asteraceae

Asteraceae

Polygonaceae

Orchidaceae

Orchidaceae

Orchidaceae

Araliaceae

Poaceae

Asteraceae

Poaceae 
Appendix 2 Taxonomically indeterminate listings

This appendix includes described taxa whose taxonomic status is uncertain and requires further investigation, and also potentially distinct plants whose taxonomic status has yet to be determined. In both instances, available information suggests that those plants listed could be under some level of threat. Definitions of categories follow those given in Appendix 1.

$\dagger$ denotes indigenous taxa found naturally outside New Zealand.

$\ddagger$ denotes an addition to this list (cf. de Lange et al. 1999).

\section{Qualifiers}

EW Extinct in the Wild

CD Conservation Dependent

DP Data Poor

RC Recovering

ST Stable

SO Secure Overseas

TO Threatened Overseas

HI Human Induced

RF Recruitment Failure

EF Extreme Fluctuations

OL One Location

PD Partial Decline

IE Island Endemic

\section{Extinct (2)}

Myosotis traversii var. cinerascens (Petrie) L.B.Moore (WELT 2585) DP

Pseudognaphalium (a) (CHR 365358; Zoo) DP

Boraginaceae

Asteraceae

\section{Acutely Threatened (54)}

\section{Nationally Critical (38)}

${ }^{\dagger}$ Botrychium aff. lunaria (CHR 289336; NW Nelson) TO

Calochilus aff. herbaceus (CHR 65825; Kaimaumau) SO, EF

$\$$ Cardamine (a) (CHR 500569; Awahokomo) $\mathrm{CD}, \mathrm{EF}, \mathrm{OL}$

C. (c) (CHR 65058; Reporoa Bog) DP

Celmisia aff. gracilenta (b) (CHR 469722; Mangaweka) $\mathrm{CD}$, OL

Craspedia (a) (CHR 511522; Clutha River) $\mathrm{HI}$, OL

C. (b) (CHR 516324; Leatham) $\mathrm{CD}$, OL

C. (i) (CHR 395643; Fyfe River) oL

C. (j) (CHR 516302; Lake Heron) HI, OL

Euphrasia (a) (CHR 471903; "white") EF, OL

$¥$ Festuca aff. novae-zelandiae (AK 252541; Awahokomo) $\mathrm{CD}$, EF, OL

Gentiana aff. astonii (a) (CHR 529112; Mt Brown)

G. aff. astonii (b) (CHR 529111; Pareora River) OL

$¥ G$. aff. astonii (c) (CHR 542276; Manahune) OL

${ }^{\ddagger}$. aff. saxosa (AK 7316; Charleston) OL

Hebe aff. bishopiana (AK 202263; Hikurangi Swamp) CD, HI, OL

$¥$ Hypsela aff. rivalis (CHR 369981; Burgoo Stream) $\mathrm{EF,} \mathrm{OL}$

Isoetes aff. kirkii (CHR 247118A; Lake Omapere) OL

$¥$ Koeleria aff. novozelandica (AK 252546; Awahokomo) $\mathrm{CD}$, OL

Lepidium aff. oleraceum (a) (AK 230459; Chatham Islands) $\mathrm{CD}$, OL

${ }^{*}$. aff. oleraceum (d) (AK 255607; Mangere) $\mathrm{EF}$, OL

Leptinella (a) (CHR 515297; Clutha River)

Limosella (b) (CHR 515038; Manutahi) ST

Linum monogynum var. chathamicum Cockayne (CHR 417633) CD, EF

$\$$ Melicytus aff. obovatus (b) (AK 235617; Mt Burnett) $\mathrm{CD}$, OL

$¥$ Microtis aff. unifolia (CHR 532775; Fox) DP, OL

Myosotis (b) (CHR 386966; Mt Tapuae-O-Uenuku) OL

Olearia aff. odorata (CHR 386084; Canterbury Plains) RF

Pachycladon aff. fastigiata (CHR 279206; Chalk Range)

Pimelea (a) (CHR 495025; Turakina) $\mathrm{CD}$, HI

$P$. aff. aridula (a) (CHR 282959; Te Mata Peak) OL

Ophioglossaceae

Orchidaceae

Brassicaceae

Brassicaceae

Asteraceae

Asteraceae

Asteraceae

Asteraceae

Asteraceae

Orobanchaceae

Poaceae

Gentianaceae

Gentianaceae

Gentianaceae

Gentianaceae

Plantaginaceae

Lobeliaceae

Isoetaceae

Poaceae

Brassicaceae

Brassicaceae

Asteraceae

Plantaginaceae

Linaceae

Violaceae

Orchidaceae

Boraginaceae

Asteraceae

Brassicaceae

Thymelaeaceae

Thymelaeaceae 
Pratia aff. angulata (AK 212143; Woodhill)

Ranunculus (a) (AKU 19876; Hope)

$R$. aff. royi (CHR 513327; Waihao) $\mathrm{HI}$, OL

Thelymitra (a) (WELT 79140; Ahipara) $\mathrm{CD}$, DP, HI, EF

Tmesipteris aff. tannensis (CHR 496779; Banks Peninsula) DP

¥Trichomanes (AK 252983; Kerikeri) DP, OL

†risetum aff. lepidum (AK 251835; Awahokomo) CD, EF, OL

\section{Nationally Endangered (14)}

Brachyscome (a) (WELT 10278; Ward)

Cardamine (b) (CHR 312947; "tarn") DP, EF

C. (d) (CHR 511706; Pisa Range) DP

†Craspedia (e) (CHR 514391; “tarn”) $\mathrm{CD}$, OL

${ }^{\ddagger}$ Gingidia aff. montana (a) (CHR 510570; Mt Burnett) $\mathrm{HI}$

$\ddagger$ Hebe aff. albicans (AK 252966; Mt Burnett) OL

Hibiscus aff. trionum (AK 218967; North Island)

Lepidium aff. oleraceum (b) (AK 208579; Antipodes) CD, DP, EF

L. aff. oleraceum (c) (CANU 5995; Snares) DP

Melicytus (a) (CHR 355077; Matiri Range)

Myosotis aff. pygmaea (CHR 244566; Volcanic Plateau) $\mathrm{CD}$

Pimelea aff. aridula (b) (AK 230900; Cook Strait) OL

Ranunculus aff. stylosus (CHR 515131; Manuhune) HI, OL

Rhopalostylis aff. sapida (AK 227148; Chatham Islands) CD, IE

3. Nationally Vulnerable (2)

${ }^{\ddagger}$ Kunzea aff. ericoides (a) (AK 255350; Thornton) $\mathrm{CD}$, RC, OL

Prasophyllum aff. patens (AK 236408; New Zealand) CD, DP

\section{Chronically Threatened (12)}

\section{Serious Decline (4)}

$\$$ Hypericum aff. japonicum (a) (CHR 165889; Volcanic Plateau) DP

Pimelea aff. arenaria (AK 216133; southern New Zealand) DP, RF

Pittosporum aff. crassifolium (AK 253259; Raoul Island) RF, OL, IE

Sicyos aff. australis (AK 252822; New Zealand)

\section{Gradual Decline (8)}

†Christella aff. dentata (b) (AK 126902; "thermal") $\mathrm{HI}$

$\$$ Craspedia (n) (CHR 369978; Henderson) OL

Eryngium aff. vesiculosum (AK 232583; New Zealand)

$¥$ Hoheria aff. sexstylosa (AK 234306; Tararua Ranges) ${ }_{\mathrm{HI}}$

Leptinella dioica subsp. monoica (AK 200874)

$¥$ Melicytus aff. alpinus (f) (CHR 541566; Waipapa)

$¥$ Ranunculus (b) (CHR 324466; Burgoo Stream)

$¥$ Raoulia aff. hookeri (AK 239529; “coast”)

\section{At Risk (89)}

\section{Sparse (14)}

Coriaria (a) (CHR 469745; Rimutaka)

ॠDianella aff. nigra (b) (AK 252911; Kopouatai) DP

\#ebe aff. diosmifolia (AK 215221; "summer flowering tetraploid”)

Hymenophyllum aff. flexuosum (AK 177370; Mount Burnett)

$¥$ Hypericum aff. japonicum (b) (CHR 140620; “tarn”) DP

¿Libertia aff. peregrinans (AK 14642; "nonoploid")

${ }^{\ddagger}$ Myosotis spathulata var. radicata L.B.Moore (CHR 87680)

$¥ M$. aff. australis (AK 231051; “small white”) DP

Myrsine aff. divaricata (AK 228797; Poor Knights)

Oreomyrrhis (CHR 364086; "minute flower")

Oxalis aff. rubens (AK 234308; "scree")

Peperomia aff. urvilleana (AK 206056; "purple vein") DP

$¥$ Raoulia (c) (CHR 401140; "M")

¥Thelymitra aff. ixioides (AK 251348; New Zealand) DP, SO, EF
Lobeliaceae

Ranunculaceae

Ranunculaceae

Orchidaceae

Psilotaceae

Hymenophyllaceae

Poaceae

Asteraceae

Brassicaceae

Brassicaceae

Asteraceae

Apiaceae

Plantaginaceae

Malvaceae

Brassicaceae

Brassicaceae

Violaceae

Boraginaceae

Thymelaeaceae

Ranunculaceae

Arecaceae

Myrtaceae

Orchidaceae

Clusiaceae

Thymelaeaceae

Pittosporaceae

Cucurbitaceae

Thelypteridaceae

Asteraceae

Apiaceae

Malvaceae

Asteraceae

Violaceae

Ranunculaceae

Asteraceae

Coriariaceae

Asparagaceae

Plantaginaceae

Hymenophyllaceae

Clusiaceae

Iridaceae

Boraginaceae

Boraginaceae

Myrsinaceae

Apiaceae

Oxalidiaceae

Piperaceae

Asteraceae

Orchidaceae 


\section{Range Restricted (75)}

$¥$ Ascarina lucida var. lanceolata (Hook.f.) Allan (AK 233919)

${ }^{\ddagger}$ Astelia aff. nervosa (a) (AK 108205; Mount Stokes) DP, OL

$¥$ A. aff. nervosa (b) (CHR 355412; Stewart Island)

$¥$ A. aff. graminea (CHR 129122; Red Hills) OL

Brachyscome aff. humilis (AK 231703; West Dome) DP, OL

$\ddagger$ Cardamine (e) (AK 231673; West Dome) DP, OL

$\$$ Carex (a) (CHR 395744; Takaka)

$\$ C$. (b) (AK 232856; Matiri)

$¥$ Celmisia aff. major (AK 255352; Pupu)

$\$$ Chionochloa (a) (OTA 18879; Mt Burns) DP

†Colobanthus (b) (AK 232645; "Red Hills")

$\$$ C. aff. wallii (AK 232551; "serpentine")

\$Coprosma aff. acerosa (AK 36799; Taranaki)

${ }^{\ddagger}$. aff. neglecta (AK 250769; Whangaroa)

Craspedia aff. minor (AK 228074; Chatham Island) IE

C. (c) (CHR 529115; Kaitorete) $\mathrm{CD}$, HI, OL

C. (d) (CHR 245893; Otakeho) DP

C. (f) (CHR 514362; Hackett) $\mathrm{OL}$

C. (g) (CHR 469764; Pikikiruna) OL

C. (h) (CHR 260312; Gouland Downs) DP

†. (k) (CHR 283173; “coast”)

$\ddagger$ C. (l) (CHR 479212; Charleston) DP, OL

$¥ C$. (o) (CHR 471883; Loveridge) DP, OL

\$C. (p) (CHR 469073; Havelock River) OL

$\ddagger$ C. (q) (AK 251905; Anglem) DP, OL

${ }^{\ddagger}$ C. (r) (CHR 313349; Punakaiki) DP, OL

$\ddagger$ C. (s) (CHR 401645; "serpentine")

$\stackrel{+}{\dagger}$. (t) (CHR 365392; Chalk)

$\$$ Deyeuxia aff. quadriseta (AK 252511; Volcanic Plateau)

$\ddagger$ Dianella aff. nigra (a) (AK 256873; Hauturu)

$¥$ Epilobium aff. glabellum (CHR 387893; "pink”) DP

‡entiana (a) (CHR 395723; Lookout Range)

G. aff. astonii (c) (CHR 519113; Awahokomo) EF, OL

G. aff. astonii (d) (CHR 529114; Ward) ${ }_{\text {ST }}$

$\ddagger G$. aff. astonii (f) (CHR 279272; Chalk Range)

$\ddagger$ G. aff. tenuifolia (CHR 387194; "stellar")

Geranium (a) (CHR 518296; Pareora River) OL

G. (b) (CHR 469918; Red Hills) ST, OL

\$Gingidia aff. montana (b) (CHR 103349; North Otago) DP

Hebe aff. ligustrifolia (AK 207101; Surville Cliffs)

$\$ H$. aff. pinguifolia (CHR 461354; "high flyer”) DP

$H$. sp. (Veronica salicifolia var. angustissima Cockayne) (AK 233637)

Helichrysum aff. aggregatum (AK 54473; Surville Cliffs) $\mathrm{CD}$, ST, OL

$¥ H$. aff. intermedium (CHR 274826; Chalk Range)

+ Lastreopsis aff. glabella (AK 242151; Kermadecs) OL, IE

$¥$ Melicytus aff. alpinus (g) (CHR 541569; Blondin)

$¥$ M. aff. crassifolius (CHR 279358; “cliff”)

$¥ M$. aff. novae-zelandiae (CHR 89907; "maritime") $\mathrm{ST}$

$\$$ M. ramiflorus subsp. (a) (AK 207155; Three Kings) DP

$¥$ M. ramiflorus subsp.(b) (AK 234207; Raoul) OL, IE

$\ddagger$ Myosotis aff. brockiei (CHR 497375; Lake Otuhie) OL

M. (a) (CHR 320240; Mossburn)

†Nephrolepis (a) (AK 232904; "thermal") TO

Notothlaspi (a) (CHR 363071; Red Hills) OL

$¥$ Ourisia aff. caespitosa (CHR 395703; Hope Range)

Parahebe aff. catarractae (CHR 324810; "hairy") DP

Phyllocladus aff. trichomanoides (AK 138493; Surville Cliffs) CD, ST, OL

Phormium aff. tenax (CHR 536735; Chatham Islands) IE

Chloranthaceae

Asteliaceae

Asteliaceae

Asteliaceae

Asteraceae

Brassicaceae

Cyperaceae

Cyperaceae

Asteraceae

Poaceae

Caryophyllaceae

Caryophyllaceae

Rubiaceae

Rubiaceae

Asteraceae

Asteraceae

Asteraceae

Asteraceae

Asteraceae

Asteraceae

Asteraceae

Asteraceae

Asteraceae

Asteraceae

Asteraceae

Asteraceae

Asteraceae

Asteraceae

Poaceae

Asparagaceae

Onagraceae

Gentianaceae

Gentianaceae

Gentianaceae

Gentianaceae

Gentianaceae

Geraniaceae

Geraniaceae

Apiaceae

Plantaginaceae

Plantaginaceae

Plantaginaceae

Asteraceae

Asteraceae

Dryopteridaceae

Violaceae

Violaceae

Violaceae

Violaceae

Violaceae

Boraginaceae

Boraginaceae

Nephrolepidaceae

Brassicaceae

Plantaginaceae

Plantaginaceae

Phyllocladaceae

Asparagaceae 
$\ddagger$ Pimelea (b) (AK 165780; Mt Manaia) $\mathrm{ST}$

$¥ P$. (c) (CHR 511713; “tarn”) DP

$\ddagger P$. (d) (CHR 472016; Pisa)

$P$. aff. aridula (c) (CHR 402249; Moawhango) ST, OL

$P$. aff. aridula (d) (CHR 221089; Maungaharuru) $\mathrm{OL}$

Pimelea aff. sericeovillosa (CHR 467766; Cobb)

$¥ P$. aff. tomentosa (b) (AK 130893; Surville Cliffs) CD, ST, OL

$¥ P$. aff. tomentosa (c) (AK 228145; Three Kings) $\mathrm{IE}$

$\$$ Pseudopanax aff. lessonii (AK 46066, Surville Cliffs)

$\$$ Pteris aff. macilenta (AK 210045; Punakaiki) DP

$\ddagger$ Raoulia aff. bryoides (AK 323119; “L”) DP, ST, OL

$\ddagger$ Rubus aff. schmidelioides (CHR 325720; “strawberry”)

\$Schizeilema (a) (CHR 190698; Ruahine)

¥Stellaria aff. parviflora (AK 169580; Poor Knights)

†Thelymitra (b) (CHR 518036; “darkie") $\mathrm{EF}$

$¥ T$. (c) (CHR 518036; “rough leaf”) $\mathrm{EF}$

\$Wahlenbergia congesta subsp. haastii J.A.Petterson (CHR 201850)

\section{Data Deficient (51)}

$¥$ Agrostis (a) (CHR 402485; Dunstan Range) OL

$\$$ Brachyglottis cockaynei (G.Simpson et J.S.Thomson) B.Nord. (AK 253995)

$\$$ B. rotundifolia var. ambigua (Cheeseman) B.Nord. (AK 251870) OL

${ }^{\ddagger}$ Cardamine aff. bilobata (CHR 511915; eastern South Island)

Carex kirkii var. elatior Kük. (CHR 73151) OL

$\$$ Celmisia cordatifolia var. brockettii Martin (WELT 4496)

C. cordatifolia var. similis Martin (CHR 365726)

C. aff. discolor (CHR 197967; Fiordland)

C. aff. gracilenta (a) (CHR 282958; Te Mata Peak) OL

Corallospartium crassicaule var. racemosum Kirk (CHR 141532)

$\$$ Dracophyllum (Venter 13745; Mt Rochfort) DP

$\$$ Elymus aff. solandri (a) (AK 222754; "channel”)

$\ddagger$ E. aff. solandri (b) (CHR 1613; South Marlborough)

¥alium aff. perpusillum (CHR 476063; Kaitorete)

$¥$ Geranium (c) (CHR 546319; Von) OL

$¥$ Grammitis aff. rawlingsii (a) (CHR 420132; Great Barrier)

$\ddagger G$. aff. rawlingsii (b) (AK 236942; Mt Williams) OL

Hebe aff. brevifolia (AK 235669; Surville Cliffs) $\mathrm{CD}$, OL

${ }^{\ddagger} H$. aff. treadwellii (CHR 394533; Bald Knob Ridge) OL

Leptinella intermedia (D.G.Lloyd) D.G.Lloyd et C.Webb (CANU 17225)

¥Libertia aff. ixioides (a) (CHR 469712; "large capsule")

Libertia aff. ixioides (b) (CHR 174779; Omaha)

Limosella (a) (CHR 222625; Opunake)

$\$$ Melicytus aff. alpinus (a) (CHR 541565; Rangipo)

${ }^{\ddagger} M$. aff. alpinus (b) (CHR 541568; Otago)

$\$ M$. aff. alpinus (c) (CHR 541567; "dark")

$¥ M$. aff. alpinus (d) (CHR 530143; "Brockie") OL

$¥ M$. aff. alpinus (e) (CHR 514919B; Livingstone)

$\$$ M. aff. obovatus (c) CHR 393733; Mt Owen) OL

$\$$ Myosotis aff. pulvinaris (CHR 431563; Umbrella)

$¥ M$. aff. tenericaulis (AK 7570; Garvie)

$\$ M$. (c) (CHR 198630; Fiordland)

¥Nematoceras aff. rivularis (CHR 534752; "rest area")

$¥ N$. aff. rivularis (CHR 518313; “whiskers”)

$¥ N$. aff. rivularis (CHR 518025; Kaimai)

$\$ N$. aff. rivularis (AK 251833; Kaitarakihi) OL

$\ddagger N$. aff. trilobus (CHR 518304; "pygmy”)

$¥ N$. aff. trilobus (CHR 537604; Rimutaka)

$¥ N$. aff. trilobus (CHR 534742; Trotters )

$¥$ Pachystegia aff. insignis (CHR 565298; Lowry)

$\$$ Pimelea aff. prostrata (CHR 257898; Kaitorete) OL

$\$ P$. (f) (AK 189577; Maunganui Bluff) OL
Thymelaeaceae

Thymelaeaceae

Thymelaeaceae

Thymelaeaceae

Thymelaeaceae

Thymelaeaceae

Thymelaeaceae

Thymelaeaceae

Araliaceae

Pteridaceae

Asteraceae

Rosaceae

Araliaceae

Caryophyllaceae

Orchidaceae

Orchidaceae

Campanulaceae

Poaceae

Asteraceae

Asteraceae

Brassicaceae

Cyperaceae

Asteraceae

Asteraceae

Asteraceae

Asteraceae

Fabaceae

Ericaceae

Poaceae

Poaceae

Rubiaceae

Geraniaceae

Grammitidaceae

Grammitidaceae

Plantaginaceae

Plantaginaceae

Asteraceae

Iridaceae

Iridaceae

Plantaginaceae

Violaceae

Violaceae

Violaceae

Violaceae

Violaceae

Violaceae

Boraginaceae

Boraginaceae

Boraginaceae

Orchidaceae

Orchidaceae

Orchidaceae

Orchidaceae

Orchidaceae

Orchidaceae

Orchidaceae

Asteraceae

Thymelaeaceae

Thymelaeaceae 
$¥$ Poa aff. sublimis (CHR 402510; Eyre Mountains) OL

$\$$ Pratia aff. macrodon (AK 255606; Old Man Range) OL

$\ddagger$ Pterostylis aff. graminea (CHR 513330; “sphagnum”)

$\$$ Ranunculus aff. reflexus (CHR 394270; Mt Peel)

$\doteqdot R$. (c) (CHR 472008; Garvie Range)

Rytidosperma tenue (Petrie) Connor et Edgar (WELT 39920)

$\ddagger$ Raoulia (a) (CHR 79537; “K”)

Spiranthes aff. novae-zelandiae (CHR 518297; Motutangi) HI, EF

$¥$ Thelymitra aff. longifolia (CHR 537579; Whakapapa)
Poaceae

Lobeliaceae

Orchidaceae

Ranunculaceae

Ranunculaceae

Poaceae

Asteraceae

Orchidaceae

Orchidaceae

Appendix 3. Taxonomically Determinate (1) and Indeterminate (2) plants previously listed by de Lange et al. (1999) or Hitchmough (2002) but which, for the reasons specified are no longer considered to be threatened.

${ }^{1}$ Ecology and distribution better understood

${ }^{2}$ Adventive

${ }^{3}$ More abundant than previously believed

${ }^{4}$ Taxonomically indistinct

${ }^{5}$ Unsupported by herbarium evidence

\section{Not Threatened (Taxonomically Determinate) (31)}

Brachyglottis traversii (F.Muell.) B.Nord. ${ }^{1}$

$\$$ Carex kaloides Petrie ${ }^{1,3}$

$\dagger$ Crassula colorata var. acuminata (Reader) Toelken ${ }^{2}$

Festuca actae Connor 1,3

${ }^{\ddagger}$ Galium trilobum Colenso ${ }^{1,3}$

Gentiana matthewsii Petrie ${ }^{4}$

Geum pusillum Petrie ${ }^{1}$

Gingidia baxterae (J.W.Dawson) C.Webb ${ }^{1,3}$

Hebe buchananii (Hook.f.) Cockayne et Allan ${ }^{1,3}$

H. colensoi (Hook.f.) Cockayne et Allan ${ }^{1,3}$

H. diosmifolia (A.Cunn.) Cockayne et Allan 1,3

H. divaricata (Cheeseman) Cockayne et Allan ${ }^{1,3}$

H. haastii (Hook.f.) Cockayne et Allan ${ }^{1,3}$

H. imbricata Cockayne et Allan ${ }^{1,3}$

H. laingii (Cockayne) Cockayne et Allan ${ }^{1,3}$

H. murrellii G.Simpson et J.S.Thomson ${ }^{1,3}$

H. propinqua (Cheeseman) Cockayne et Allan ${ }^{1,3}$

H. rupicola (Cheeseman) Cockayne et Allan ${ }^{1,3}$

H. strictissima (Kirk) L.B.Moore ${ }^{1,3}$

Ileostylus micranthus (Hook.f.) Tiegh. ${ }^{3}$

Lachnagrostis elata Edgar ${ }^{1,3}$

L. littoralis subsp. salaria Edgar ${ }^{1,3}$

Libertia edgariae Blanchon, Braggins et B.G.Murray ${ }^{1,3}$

${ }^{\dagger}$ Limosella curdieana F.Muell. ${ }^{2}$

Olearia bullata H.D.Wilson et Garn.-Jones 1,3

Parahebe catarractae subsp. lanceolata (Benth.) Garn.-Jones 1, 3

Polystichum silvaticum (Colenso) Diels ${ }^{1,3}$

Ranunculus berggrenii Petrie $^{4}$

$¥$ Raoulia parkii Buchanan ${ }^{3}$

Trichomanes strictum Menzies, Hook. et Grev. ${ }^{1,3}$

Veronica irrigans Kirk $^{4}$

2. Not Threatened (Taxonomically Indeterminate) (18)

Brachyscome (b) (CHR 518295; Pareora River) ${ }^{4}$

Celmisia aff. sessiliflora (CHR; Bald Knob Ridge) ${ }^{5}$

Colobanthus (a) (CHR 515133; Pareora River) ${ }^{4}$

Cortaderia aff. fulvida (CHR 477325; Puketi) ${ }^{4}$

Cunoniaceae genus indeterminate (AK 230695; Te Urewera) ${ }^{4}$

Dracophyllum pearsonii $\mathrm{Kirk}^{1,3}$

D. aff. traversii (Venter 13827; "gabbro")1,3

Gentiana (b) (AK 232641; Red Hills) ${ }^{4}$
Asteraceae

Cyperaeae

Crassulaceae

Poaceae

Rubiaceae

Gentianaceae

Rosaceae

Apiaceae

Plantaginaceae

Plantaginaceae

Plantaginaceae

Plantaginaceae

Plantaginaceae

Plantaginaceae

Plantaginaceae

Plantaginaceae

Plantaginaceae

Plantaginaceae

Plantaginaceae

Loranthaceae

Poaceae

Poaceae

Iridaceae

Scrophulariaceae

Asteraceae

Plantaginaceae

Dryopteridaceae

Ranunculaceae

Asteraceae

Hymenophyllaceae

Plantaginaceae

Asteraceae

Asteraceae

Caryophyllaceae

Poaceae

Cunoniaceae

Ericaceae

Ericaceae

Gentianaceae 
Hebe pimeleoides var. glauco-caerula (J.B.Armstr.)

Plantaginaceae

Cockayne et Allan (CHR 462377) ${ }^{4}$

$H$. aff. epacridea (CHR 470336; Mt Dobson) ${ }^{4}$

${ }^{\ddagger}$. aff. macrocarpa var. latisepala (AK 250801; Great Barrier Island) ${ }^{4}$

$¥$ Hebe aff. pimeleoides (CHR 173403; Mackenzie Basin) ${ }^{4}$

Hypoxis aff. hookeri (CHR 486447; New Zealand) ${ }^{2}$

Kunzea aff. ericoides (AK; "sand") 1,3

Melicytus aff. alpinus (a) (AK 230926; Wairarapa) ${ }^{4}$

Melicytus aff. obovatus (a) (AK 229988; Cook Strait) ${ }^{1,3}$

Ourisia aff. macrocarpa (CHR; Richmond Range) $)^{5}$

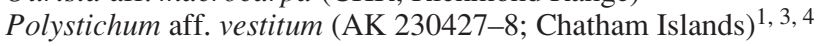

Plantaginaceae

Plantaginaceae

Plantaginaceae

Hypoxidaceae

Myrtaceae

Violaceae

Violaceae

Plantaginaceae

Dryopteridaceae

Appendix 4 Nomenclature changes affecting taxa listed by de Lange et al. (1999) and Hitchmough (2002).

\section{Previous listings}

Abrotanella patearoana Heads

Ackama (AK 253151; Waima Forest)

Astelia aff. nervosa (a) (CHR; Mt Stokes)

Astelia aff. nervosa (b) (CHR; Stewart Island)

A. graminea (CHR; Red Hills)

Atriplex aff. billardierei (AK 225956; North Island)

Caleana minor R.Br.

Cardamine (a) (CHR 312947; "tarn")

Cardamine (c) (CHR 500569; Awahokomo)

Cardamine (b) (CHR 511706; Pisa Range)

Cardamine (d) (AK; West Dome)

Carex (a) (CHR; Takaka)

Carex (b) (CHR; Matiri)

Caladenia atradenia D.L.Jones, Molloy et M.A.Clem.

Cheesemania enysii (Cheeseman) O.E.Schulz

Cheesemania fastigiata (Hook.f.) O.E.Schulz

Cheesemania stellata (Allan) Heenan

Cheesemania aff. fastigiata (CHR 279206; Chalk Range)

Chiloglottis formicifera Fitz. pro parte*

Chiloglottis valida D.L.Jones

Chionochloa aff. macra (CHR; Mt Burns)

Christella aff. dentata (a) (CHR 472870; Kaitaia)

Colobanthus (b) (CHR; Red Hills)

C. aff. wallii (CHR; "serpentine")

Coprosma aff. acerosa (CHR; Taranaki)

Coprosma aff. neglecta (AK; Whangaroa)

Coprosma aff. obconica (AK 36254; Surville Cliffs)

Coprosma aff. spathulata (AK 229538; Surville Cliffs)

Corunastylis nudum (Hook.f.) D.L.Jones et M.A.Clem Corunastylis nudum (Hook.f.) D.L.Jones et M.A.Clem Corybas carsei (Cheeseman) Hatch

Craspedia (k) (CHR; "coast")

Craspedia (1) (CHR; Charleston)

Craspedia (o) (CHR; Loveridge)

Craspedia (p) ( CHR; Havelock

Craspedia (q) (CHR; Anglem)

Craspedia (r) (CHR; Punakaiki)

Craspedia s (CHR; "serpentine")

Craspedia (t) (CHR; Chalk)

Dianella aff. nigra (a) (CHR; Hauturu)

Dianella aff. nigra (b) (CHR; Kopouatai)

\section{This paper}

Abrotanella patearoa Heads

Ackama nubicola de Lange

Astelia aff. nervosa (a) (AK 108205; Mount Stokes)

Astelia aff. nervosa (b) (CHR 355412; Stewart Island)

Astelia aff. graminea (CHR 129122; Red Hills)

Atriplex hollowayi de Lange et D.A.Norton

Paracaleana minor (R.Br.) Blaxell

Cardamine (b) (CHR 312947; "tarn")

Cardamine (a) (CHR 500569; Awahokomo)

Cardamine (d) (CHR 511706; Pisa Range)

Cardamine (e) (AK 231673; West Dome)

Carex (a) (CHR 395744; Takaka)

Carex (b) (AK 232856; Matiri)

Stegostyla atradenia (D.L.Jones, Molloy et M.A.Clem.) D.L.Jones et M.A.Clem.

Pachycladon enysii (Cheeseman) Heenan et A.Mitch.

Pachycladon fastigiata (Hook.f.) O.E.Schulz

Pachycladon stellata (Allan) Heenan et A.Mitch.

Pachycladon aff. fastigiata (CHR 279206;

Chalk Range)

Chiloglottis trapeziformis Fitz.

Simpliglottis valida (D.L.Jones) D.L.Szlachekto

Chionochloa (a) (OTA 18879; Mt Burns)

Christella dentata sens. str.

Colobanthus (b) (AK 232645; "Red Hills")

Colobanthus aff. wallii (AK 232551; "serpentine")

Coprosma aff. acerosa (AK 36799; Taranaki)

Coprosma aff. neglecta (AK 250769; Whangaroa)

Coprosma distantia (de Lange et R.O.Gardner)

de Lange

Coprosma spathulata subsp. hikuruana de Lange et Heenan

Corunastylis nuda (Hook.f.) D.L.Jones et M.A.Clem

Corunastylis pumila (Hook.f.) D.L.Jones et M.A.Clem

Anzybas carsei (Cheeseman) D.L.Jones et M.A.Clem.

Craspedia (k) (CHR 283173; “coast”)

Craspedia (1) (CHR 479212; Charleston)

Craspedia (o) (CHR 471883; Loveridge)

Craspedia (p) (CHR 469073; Havelock River)

Craspedia (g) (AK 251905; Anglem)

Craspedia (r) (CHR 313349; Punakaiki)

Craspedia (s) (CHR 401645; "serpentine")

Craspedia (t) (CHR 365392; Chalk)

Dianella aff. nigra (a) (AK 256873; Hauturu)

Dianella aff. nigra (b) (AK 252911; Kopouatai) 
Epilobium aff. glabellum (CHR; "pink")

Eryngium vesiculosum Labill

Euchiton nitidulus (Hook.f.) Anderb.

Gentianella bellidifolia var. magnifica (Kirk)

Hitchmough nom. nud.

Gentianella (a) (CHR; Lookout Range)

Geranium (c) (CHR; Von)

Gingidia aff. montana (a) (CHR; North Otago)

Hebe pimeleioides var. rupestris Cockayne et Allan

Hebe aff. bollonsii (AK 150628; Mokohinau Islands)

Hebe aff. diosmifolia (AK 215221; "summer flowering")

Hebe. aff. macrocarpa var. latisepala (AK 250801;

Great Barrier Island)

Hebe aff. pinguifolia (CHR; "high flyer")

Hebe aff. rigidula (WAIK 5822; Awaroa Valley)

Hebe aff. rigidula (CHR 192300; D'Urville Island)

$H$. sp. (Veronica salicifolia var. angustissima Cockayne)

Helichrysum aff. intermedium (CHR; Chalk Range)

Hoheria aff. sexstylosa (CHR; Tararua Ranges)

Hypericum aff. japonicum (b) (CHR; "tarn")

Ischnocarpus exilis Heenan

I. novae-zelandiae (Hook.f.) O.E.Schulz

Leucopogon aff. parviflorus (AK 130914; Surville Cliffs)

Libertia aff. grandiflora (CHR; Eastbourne)

Libertia aff. ixioides (a) (CHR; East Cape)

Libertia aff. ixioides (b) (CHR; Omaha)

Libertia aff. peregrinans (CHR; "nonoploid")

Myosotis aff. australis (CHR; "small white")

Myosotis aff. brockiei (CHR; Lake Otuhie)

Olearia (a) (AK 178700; Hauturu Range)

Oxalis aff. rubens (CHR; "scree")

Pachystegia aff. insignis (CHR; Lowry)

Pimelea (b) AK; Mt Manaia)

Pimelea (c) (CHR; "tarn")

Pimelea "Pisa" (CHR)

Pimelea aff. sericeovillosa (CHR; Cobb)

Pimelea aff. tomentosa (b) (CHR; Surville Cliffs)

Pimelea aff. tomentosa (c) (CHR; Three Kings)

Pseudopanax aff. lessonii (CHR; Surville Cliffs)

Pteris aff. macilenta (CHR; "Punakaiki")

Polystichum aff. vestitum (AK 230427-8; Chatham Islands)

Pterostylis alveata Garnet

Pterostylis banksii var. silvicultrix F.Muell.

Pterostylis puberula Hook.f.

Pterostylis tanypoda D.L.Jones, Molloy et M.A.Clem.

Pterostylis tasmanica D.L.Jones

Pterostylis tristis Colenso

Pterostylis aff. montana (CHR 534754; Catlins)

Ranunculus (b) (CHR; Burgoo Stream)

Raoulia (c) (CHR; "M")

Rubus aff. schmidelioides (AK; "strawberry")

Stellaria aff. parviflora (AK; Poor Knights)

Thelymitra aff. ixioides (CHR; New Zealand)
Epilobium aff. glabellum (CHR 387893; "pink")

Erynigium aff. vesiculosum (AK 232583;

New Zealand)

Argyrotegium nitidulum (Hook.f.) J.M.Ward et Breitw.

Gentiana bellidifolia var. magnifica Kirk

Gentiana (a) (CHR 395723; Lookout Range)

Geranium (c) (CHR 546319; Von)

Gingidia aff. montana (b) (CHR 103349; North Otago)

Hebe pimeleioides subsp. faucicola Kellow et Bayly

Hebe pubescens subsp. sejuncta Bayly et de Lange

Hebe aff. diosmifolia (AK 215221; "tetraploid")

Hebe macrocarpa (Vahl.) Cockayne et Allan var. macrocarpa

Hebe aff. pinguifolia (CHR 461354; "high flyer")

Hebe scopulorum Bayly, de Lange et Garn.-Jones

Hebe rigidula var. sulcata Bayly et Kellow

$H$. sp. (Veronica salicifolia var. angustissima Cockayne)

(AK 233637)

Helichrysum aff. intermedium (CHR 274826;

Chalk Range)

Hoheria aff. sexstylosa (AK 234306; Tararua Ranges)

Hypericum aff. japonicum (b) (CHR 140620; "tarn")

Pachycladon exilis (Heenan) Heenan et A.Mitch.

Pachycladon cheesemanii Heenan et A.Mitch.

Leucopogon xerampelinus de Lange,

Heenan et M.I.Dawson

Libertia edgariae Blanchon, Braggins et B.G.Murray

Libertia cranwelliae Blanchon, Braggins et B.G.Murray

Libertia aff. ixioides (b) (CHR 174779; Omaha)

Libertia aff. peregrinans (AK 14642; "nonoploid")

Myosotis aff. australis (AK 231051; "small white")

Myosotis aff. brockiei (CHR 497375; Lake Otuhie)

Olearia crebra E.K.Cameron et Heenan

Oxalis aff. rubens (AK 234308; "scree")

Pachystegia aff. insignis (CHR 565298; Lowry)

Pimelea (b) (AK 165780; Mt Manaia)

Pimelea (c) (CHR 511713; "tarn")

Pimelea (d) (CHR 472016; Pisa)

Pimelea aff. sericeovillosa (CHR 467766; Cobb)

Pimelea aff. tomentosa (b) (AK 130893; Surville Cliffs)

Pimelea aff. tomentosa (c) (AK 228145; Three Kings)

Pseudopanax aff. lessonii (AK 46066; Surville Cliffs)

Pteris aff. macilenta (AK 210045; Punakaiki)

Polystichum vestitum (G.Forst.) C.Presl

Diplodium alveatum (Garnet) D.L.Jones et M.A.Clem.

Pterostylis silvicultrix (F.Muell.) Molloy,

D.L.Jones et M.A.Clem.

Linguella puberula (Hook.f.) D.L.Jones, M.A.Clem. et Molloy

Hymenochilus tanypoda (D.L.Jones, Molloy

et M.A.Clem.) D.L.Jones, M.A.Clem. et Molloy

Plumatochilos tasmanicum (D.L.Jones) D.L.Szlachetko

Hymenochilus tristis (Colenso) D.L.Jones, M.A.Clem. et Molloy

Pterostylis auriculata Colenso

Ranunculus (b) (CHR 324466; Burgoo Stream)

Raoulia (c) (CHR 401140; "M")

Rubus aff. schmidelioides (CHR 325720; "strawberry")

Stellaria aff. parviflora (AK 169580; Poor Knights)

Thelymitra aff. ixioides (AK 251348; New Zealand 
Thelymitra (b) (CHR; "darkie")

Thelymitra (b) (CHR 518036; "darkie")

Thelymitra (c) (CHR; "rough leaf")

Utricularia protrusa Hook.f.

Thelymitra (c) (CHR 518036; "rough leaf")

Utricularia australis R.Br.

*The exact status of specimens of Chiloglottis, collected by R. H. Matthews near Kaitaia between 1901 and 1914, and attributed to C. formicifera Fitz. by Cheeseman (1901) and Moore (in Moore \& Edgar 1970) has been problematic. Although these specimens have many features of $C$. formicifera, they are more similar to $C$. trapeziformis Fitz. where they were placed by Molloy (in de Lange \& Murray 2002). Recently this decision has been questioned (Scanlen 2003). On the latest advice, the Australian authority on the genus, D. L. Jones (pers. comm.) who has examined Matthews' material, has placed these specimens within C. trapeziformis. It should also be noted that bona fide $C$. trapeziformis is known from New Zealand based on recent gatherings made near Hokio beach during 2001 (de Lange \& Murray 2002). 